\title{
Eine Antwort auf die «Krise der Medizin»? Die Moderne Konstitutionslehre im Krisendiskurs 1925 bis $1933^{*}$
}

Nadine Metzger

\section{Summary}

Discussing the alleged «crisis of medicine» (ca. 1925-33) German physicians addressed the underlying mindset of medical theory and practice. Identified as «scientific crisis», they called the scientific epistemology of medicine into question. Since 1911 modern constitutional medicine had offered concepts potentially useful to overcome this «crisis». Besides similar topics the two discussions shared prominent voices, presenting their ideas for better medicine in both discourses. However, this paper shows the minor role constitutional medicine actually played in the «crisis» discussion. It was rarely put forward as a solution to the supposed «crisis» and is conspicuously often discovered in discussions of historical background. Far more often, concepts designed to overcome constitutional medicine are invoked as a potential crisis solution, first and foremost the so-called «constitutional therapy» fathered by Vienna gynaecologist Bernhard Aschner which evokes pre-modern humoral pathology.

Keywords: Bernhard Aschner, crisis of medicine, constitutional medicine, holism, medical epistemology

* Mein Dank gilt den anonymen Gutachter*innen des Gesnerus für ihre wertvollen Hinweise und Kritik sowie den Teilnehmenden am Regensburger Wissenschaftshistorischen Kolloquium, insbesondere Marcus Hahn, für ihre Anregungen in einem früheren Stadium der vorliegenden Arbeit.

Nadine Metzger, Institut für Geschichte und Ethik der Medizin, Friedrich-Alexander-Universität Erlangen-Nürnberg, Glückstr. 10, 91054 Erlangen, Deutschland, Tel.: 0049-9131-85-22851, Fax: 0049-9131-85-22852, nadine.metzger@fau.de 


\section{Zusammenfassung}

Im Diskurs um die «Krise der Medizin» ca. 1925 bis 1933 stellten Ärzte unter anderem die Frage nach der grundlegenden Ausrichtung der Medizin und problematisierten in diesem Zusammenhang ihre Forschungsepisteme unter dem Schlagwort der «wissenschaftlichen Krise». Bereits seit 1911 hatte sich die sogenannte «Moderne Konstitutionslehre» mit ähnlichen Fragen zur Zukunft der Medizin beschäftigt. Neben inhaltlichen Überschneidungen teilten die beiden Diskurse auch prominente Beiträger, die in beiden Kontexten Medizinkritik anbrachten und Reformvorschläge formulierten. Trotz dieser Gemeinsamkeiten spielte die Konstitutionslehre im Krisendiskurs allerdings nur eine untergeordnete Rolle und wurde kaum als Lösungsvorschlag diskutiert. Sie wird hingegen auffallend häufig in historischen Einordnungen der «Krise» erwähnt. Weitaus mehr Erneuerungspotenzial sprachen Beiträger zum Krisendiskurs Ansätzen zu, die die Moderne Konstitutionslehre überwinden wollten, allen voran der an vormoderner Humoralpathologie orientierten «Konstitutionstherapie» des Wiener Gynäkologen Bernhard Aschner.

Schlagworte: Bernhard Aschner, Krise der Medizin, Moderne Konstitutionslehre, Holismus, Forschungsepisteme der Medizin

\section{Einleitung}

«Bleiben die Verhältnisse so wie sie sind, so ist, wie es scheint, das Schicksal der deutschen Ärzteschaft besiegelt», schrieb der Berliner praktische Arzt Ernst Mayer 1924 in «Die Krisis des deutschen Ärztestandes» und läutete damit den Diskurs um die «Krise der Medizin» ein, der besonders intensiv zwischen 1926 und 1930 innerhalb und außerhalb der medizinischen Fachblätter geführt wurde. ${ }^{1}$ Doch Rettung schien in Sicht - behauptete zumindest 1928 der Wiener Frauenarzt Bernhard Aschner in Gestalt eines von ihm selbst entwickelten therapeutischen Ansatzes. ${ }^{2}$ Sein Buch versprach bereits im Titel, Die Krise der Medizin - Konstitutionstherapie als Ausweg, vollmundig ein Ende der vielfach gepflegten Untergangsstimmung und wurde tatsächlich im Krisendiskurs häufig und ausgesprochen positiv rezipiert.

Aschners «Konstitutionstherapie» steht in Verbindung mit in den 1920er Jahren ubiquitär thematisierten Konzepten von «Konstitution», die trotz

1 Mayer 1924, 46. Mayer (1883-1952) war niedergelassener Arzt in Berlin. Seine Schwester war mit Karl Jaspers verheiratet. Zu Mayer siehe Geiger 2010.

2 «Konstitutionstherapie [...] scheint mir der beste Ausweg aus der gegenwärtigen Krise der Medizin.» Aschner 1928, 35. Näheres zu Aschner siehe unten. 
ganz unterschiedlicher Ausgestaltungen im Kern körperinhärente Faktoren für Krankheitsentstehung und -verläufe meinten, die dem einzelnen Patienten eigen sind. Als «Konstitutionslehre» oder «Konstitutionspathologie» ${ }^{3}$ hatte sich bereits vor dem Ersten Weltkrieg eine aufstrebende interdisziplinäre Forschungsrichtung formiert, die eine Wende hin zum individuellen Patienten proklamierte: Der Einzelne mit seinen spezifischen körperlichen Voraussetzungen sollte wieder in den Fokus von medizinischer Forschung und Praxis rücken. Damit positionierte sich die Konstitutionslehre explizit als Gegenbewegung zu Bakteriologie und zunehmend kritisierter «Laborwissenschaft», sie trat an, «die Medizin» aus einer vermeintlichen Sackgasse herauszuführen und grundlegend zu erneuern. Um sich vom vormodernen humoralpathologischen Konstitutionsbegriff abzugrenzen, nannte man sich «Moderne Konstitutionslehre»; methodisch war diese zunächst streng auf das naturwissenschaftliche Medizinkonzept ausgerichtet. ${ }^{4}$

Wegen ihres beträchtlichen Impetus, die Medizin in Wissenschaft und Praxis zu reformieren, wurden im Kontext der Konstitutionslehre von Beginn an medizinkritische Fragen verhandelt und in programmatischen Veröffentlichungen Aufgaben, Zielrichtung und Episteme «der Medizin» diskutiert. Verschiedene Punkte dieser Kritik kehrten in der Diskussion um die «Krise der Medizin» seit 1924 wieder, die sich nicht nur mit der wirtschaftlichen und standespolitischen Situation von Ärzten beschäftigte, sondern auch eine vorgebliche «wissenschaftliche Krise» thematisierte. So beklagten die Beiträger zum Krisendiskurs genau wie Konstitutionspathologen in den fünfzehn Jahren davor unter anderem die Patientenferne einer abgehobenen Labormedizin, lokalistische Krankheitsanschauungen und Reduktion komplexer Körperzusammenhänge auf schematische Mechanismen.

Die Konstitutionslehre bot nicht nur ein Konzept an, wie die Medizin ihren Blick auf den individuellen kranken Menschen richten könne, sondern startete auch spätestens ab 1911 mit großem Enthusiasmus in ihr interdisziplinäres Forschungsprogramm, das die Medizin als Ganzes auf eine neue Grundlage stellen wollte. Manche Autoren wiesen der Konstitutionslehre gar den Status einer grundlegenden medizinischen Betrachtungsweise zu,

3 Dieser Aufsatz benutzt beide Begriffe synonym; entsprechend werden «konstitutionspathologisch» als Adjektiv und «Konstitutionspathologe» als Personenbezeichnung verwendet. «Konstitutionsforschung» betont die Forschung zu Fragestellungen der Konstitutionslehre.

4 Zur Konstitutionslehre der 1910er und 20er Jahre siehe Metzger 2017 und 2016; Timmermann 2001 und 1996; Probst 1982. Aus Perspektive der Pathologie Prüll 1998 und 2003, insbes. 343-49; in Bezug auf Vererbungslehre und Eugenik Baader 2007, insbes. 86-92; Hofer 2007; Weindling 1989, 170-75, 232-35; in Bezug auf Eugenik und Lebensreform Hau 2003, 101-75. Als holistische Strömung Felsch 2005; Hau 2000; Prüll 1998. In Bezug auf das Problem des Individuellen in der Medizin Gadebusch Bondio/Michl 2012, insbes. 122-31; Gadebusch Bondio 2015, 24-37. 
auf einer Ebene und vergleichbar mit der Zellularpathologie als wissenschaftlichem Paradigma. ${ }^{5}$ Doch konnte die Konstitutionslehre auch noch im Krisendiskurs ab Mitte der 1920er Jahre als Antwort firmieren, fünfzehn Jahre später und in einer gänzlich veränderten Situation? Wurde ihr noch Potenzial zur Überwindung der diagnostizierten «Krise» zugestanden? Wie spielt der Erfolg von Bernhard Aschners «Konstitutionstherapie» in diese Frage hinein? In diesem Beitrag sollen deshalb die inhaltlichen und personellen Verbindungen zwischen Konstitutionslehre und Krisendiskurs 1925 bis 1933 genauer bestimmt und die Rolle der Konstitutionslehre im Krisendiskurs herausgearbeitet werden.

\section{Forschungsstand und Vorgehen}

Die Sekundärliteratur sieht insbesondere dann eine Verbindung zwischen Konstitutionslehre und Krisendiskurs, wenn sie sich mit übergreifenden intellektuellen Strömungen der 1920er Jahre befasst, insbesondere dem Holismus, der auf beiden seinen Stempel hinterlassen hat. ${ }^{6}$ Speziell der Krise der Medizin gewidmete Studien erwähnen entweder die Konstitutionslehre gar nicht, ${ }^{7}$ oder aber heben hervor, die Konstitutionslehre sei als Lösungsvorschlag im Krisendiskurs gehandelt worden. ${ }^{8}$ Letztere These wird von den Autoren zumeist als selbstevident präsentiert.

Den Beginn machte Eva Klasen in ihrer grundlegenden Dissertation zur Krise der Medizin (1984): Im Kapitel III, «Lösungsvorschläge», diskutiert sie «Konstitutionspathologie und -therapie» als einen von vier Punkten..$^{9} \mathrm{Im}$ Kapitel führt sie allerdings nur Bernhard Aschner mit seiner Konstitutionstherapie als konkretes Beispiel für einen Lösungsvorschlag aus.

Carsten Timmermann, dessen Arbeit zentral ist für das gegenwärtige Bild der Konstitutionslehre in der medizinhistorischen Forschung, zieht die gleiche Verbindung in mehreren Veröffentlichungen $;{ }^{10}$ sie hängt dabei direkt mit seiner Gesamtargumentation bezüglich Konstitutionslehre und holistischen Strömungen in den 1920er Jahren zusammen. Timmermann unter-

5 Etwa Diepgen 1928: «grundlegende Betrachtungsweise», und Brugsch 1922, 1083: «die Matrix aller medizinischen Disziplinen».

6 Hau 2000; 2004.

7 Roelcke 2016; Schmiedebach 1989; 2014; Geiger 2010; Bothe 1991.

8 Hofer 2007; Timmermann 2001; 1999; 1996; Klasen 1984.

9 Die anderen sind «Der Arzt als Biologe, Eugeniker und Rassenhygieniker», «Die Aufwertung der Bedeutung seelischer Faktoren; Psychotherapie und Psychosomatik» und «Wiederentdeckung der hippokratischen Medizin».

10 Timmermann: MA thesis 1996; PhD thesis 1999; Aufsatz im Bulletin of the History of Medicine 2001. 
scheidet seit seiner Masterarbeit 1996 zwei Richtungen in der Konstitutionslehre, die rationalistischen Konstitutionsforscher, die sich ausschließlich an naturwissenschaftlichen Methoden orientierten, und die Neo-Romantiker, deren Spielart der Konstitutionslehre durch Holismus, Anti-Mechanismus und die Betonung der Intuition geprägt worden sei. ${ }^{11}$ Die Ausrichtung dieser neo-romantischen Konstitutionspathologie habe sich nicht nur in enger Verknüpfung mit ähnlichen Ansätzen des Krisendiskurses herausgebildet, sondern ihre Vertreter brachten ihre Konstitutionslehre auch wiederum bedeutsam in den Diskurs ein:

«Authors whom I call the neo-romantics [...] offered constitutional medicine as a holist solution to the crisis they believed to be caused by the fragmentation of the patient's body by cellular pathology, as well as the fragmentation of the medical profession by specialization in modern medicine.»12

So wie Klasen nennt auch Timmermann lediglich Bernhard Aschner als explizites Beispiel für diese These. Welche anderen «Neo-Romantiker» Konstitutionsmedizin als Lösungsvorschlag in den Krisendiskurs eingebracht haben, bleibt in seinen Veröffentlichungen zum Thema vage. ${ }^{13}$ Veronika Hofer übernimmt die These in ihrer Arbeit zum Wiener Konstitutionspathologen Julius Bauer von 2007 und generalisiert, Konstitutionslehre sei «ein Versuch [...] auf die Krise der Medizin zu antworten». ${ }^{14}$

Die These von der Konstitutionslehre als Lösungsvorschlag im Diskurs um eine «Krise der Medizin» ist somit in der Sekundärliteratur zu Konstitutionslehre und Krisendiskurs stark präsent. ${ }^{15}$ Wie diese Arbeit zeigen

11 Timmermann 1996, 14.

12 Timmermann 1996, 10. Außerdem Timmermann 1999, 55: «Constitutional research was extremely popular amongst medical researchers, and many believed that it may provide potential solutions to the crisis of medicine by overcoming the fragmentation of the body and of medical science.» Sowie Timmermann 2001, 737: «Neoromantics presented Konstitutionslehre to the public as a potential solution for what they saw as a 'crisis of medicine'.»

13 Timmermann nennt zwar verschiedene «neo-romantische» Beiträger zum Krisendiskurs, insbesondere Erwin Liek, August Bier und Hans Much, verliert aber kein Wort über deren Haltung zur Konstitutionslehre. Lediglich Liek wird einmal als «another prominent advocate of neoromantic constitutional concepts» (Timmermann 2001,721) bezeichnet, ohne dies näher zu erläutern. Umgekehrt werden namentlich genannte «neo-romantische» Konstitutionspathologen (Kraus, Brugsch und von Bergmann bei Timmermann 1996, 12, vgl. differenzierter Timmermann 2001, 725-726) nicht mit dem Krisendiskurs verbunden. Alle in dieser Fußnote genannten Autoren außer von Bergmann werden unten näher besprochen.

14 Hofer 2007, 43.

15 Nicht erwähnt wird die These von Bothe 1991, auch nicht in seinem Kapitel «Vorschläge zur Überwindung der Krise der Medizin» (S. 31-37), sowie in den Arbeiten von Schmiedebach zur Krise der Medizin. Schmiedebach 2014, 122, spricht zwar von einer «Unzahl von Lösungsvorschlägen», bei den Beispielen für ebensolche ist die Konstitutionslehre aber nicht aufgeführt. Volker Roelcke 2016 fokussiert auf Psychotherapie und Alternativmedizin. Karin Geiger 2010 stellt andere Fragen. Michael Hau beschäftigt sich in seinen Arbeiten hauptsächlich mit Holismus, daher ist es für ihn unwichtig, Konstitutionslehre und Krisendiskurs streng voneinander abzugrenzen, da in beiden Holismus verhandelt wird (Hau 
wird, ist sie aber nicht in der Lage, die Rolle der Konstitutionslehre im Krisendiskurs adäquat zu beschreiben.

Im Folgenden werde ich zunächst die inhaltlichen Überschneidungen der medizinkritischen Äußerungen im Kontext der Modernen Konstitutionslehre ab 1911 und innerhalb des Krisendiskurses 1925 bis 1933 sowie ihre Unterschiede herausarbeiten. Dann soll zuerst die Position von Aschners Konstitutionstherapie sowohl in Krisendiskurs als auch in Bezug auf die Moderne Konstitutionslehre geklärt werden, bevor die verschiedenen Rollen analysiert werden, die der Konstitutionslehre im Krisendiskurs zugewiesen werden. Zuletzt werden mit den Beiträgern zu Konstitutionslehre und Krisendiskurs personelle Verschränkungen aufgezeigt und eingeordnet, bevor im Fazit einige Folgerungen zur Bedeutung der Konstitutionslehre innerhalb des Krisendiskurses sowie ihrer Entwicklung als ehemaliger Hoffnungsträgerin angeschlossen werden.

Um die inhaltlichen und personellen Parallelen zwischen Konstitutionslehre und Krisendiskurs zu klären, betrachte ich beide als voneinander abgrenzbare Diskurse. Die eindeutige Zuordnung wird beim Krisendiskurs dadurch erleichtert, dass die Autoren ihre Beiträge fast immer selbst unter das Schlagwort «Krise» oder «Krisis» der Medizin stellten. ${ }^{16}$ Für die Konstitutionslehre betrachte ich medizinreflektierende Beiträge und Äußerungen, deren Autoren diese, meist durch Überschrift oder auch klare Verortung im Text, ebenfalls selbst der Konstitutionslehre zuordnen.

Um die Rolle der Konstitutionslehre im Krisendiskurs zu bestimmen, habe ich 52 Veröffentlichungen desselben ausgewertet. In ihrer Zahl enthalten sind einerseits sämtliche Quellen, die von Klasen, Timmermann und Bothe in ihren jeweiligen einschlägigen Kapiteln zitiert wurden, sowie dort nicht aufgeführte explizite Beiträge zur «wissenschaftlichen Krise» aus den Jahren 1925 bis 1933, andererseits Äußerungen bekannter Konstitutionspathologen zum Krisendiskurs im gleichen Zeitraum. Die überwiegende Anzahl dieser Arbeiten beschäftigt sich dezidiert mit der «wissenschaftlichen Krise», allerdings erwähnen nur 16 davon die Konstitutionslehre oder überhaupt das Stichwort «Konstitution» (ausgenommen Konstitutionstherapie). ${ }^{17}$ Alle diese Beiträge werden unten behandelt.

2000); die Lösungsvorschlags-These kommt bei ihm nicht vor. Michael Knipper 2007 nennt Aschner beispielhaft als einen Autor, der die Krise «für spezifische Partikulärzwecke instrumentalisiert» (S. 391), einer Einschätzung, der ich mich anschließe.

16 Fruchtbar wird der Begriff des Diskurses für die Diskussion um die «Krise der Medizin» bereits von Geiger 2010 in ihrem methodisch sehr reflektierten Artikel verwendet, der unter anderem den Konstruktcharakter der «Krise» herausarbeitet.

17 Krehl 1926; Sauerbruch 1926; Liek 1926; Goldscheider 1927; Honigmann 1927; Honigmann 1928a; Brugsch 1928; Diepgen 1928; Grote 1928; Bumke 1929; Sigerist 1930; His 1930; Much 1931; Bier 1931; Gruber 1933. Huismans 1929 nennt zwar das Schlagwort der Krise 


\section{Medizinkritik in den Diskursen um Konstitution und Krise}

Als 1911 auf dem 28. Deutschen Kongress für innere Medizin in Wiesbaden das Thema «Konstitution» vor großem Publikum und mit beträchtlichem Nachhall verhandelt wurde, forderten die Hauptredner eine wissenschaftliche Bearbeitung des zugrundeliegenden Phänomens. Sie stellten dabei die Grundzüge eines Forschungsprogrammes auf, das individuelle Krankheitsneigung methodisch reflektiert und naturwissenschaftlich orientiert aufklären sollte. Dass Menschen unterschiedlich auf Krankheitsreize reagierten, sei allseits beobachtbar - nun sollte sich auch die medizinische Forschung mit Konstitution beschäftigen und damit den Blick auf den einzelnen Patienten in seiner körperlichen Individualität richten, im Dienste einer patientenzugewandten, individualisierten klinischen Praxis. ${ }^{18}$

Zurückgegriffen wurde dabei unter anderem auf Vorarbeiten von Friedrich Martius (1850-1923), der seit der Jahrhundertwende für die Wiedereinführung des Konzeptes der Konstitution in das medizinische Lehrgebäude gestritten hatte. ${ }^{19}$ Der Internist Martius, Ordinarius in Rostock, positionierte seine konstitutionspathologische Perspektive vehement gegen eine Überschätzung äußerer Krankheitsauslöser, wie sie seiner Meinung nach die «orthodoxe Bakteriologie $»^{20}$ praktizierte: Bitter beklagte er die Vormachtstellung, die der «Bakteriologismus» in Fachpolitik und Krankheitsdeutung innehabe. ${ }^{21}$ Die Konstitutionslehre solle die «Überspannung der rein experimentellen Ära $»^{22}$ überwinden und die Medizin ans Krankenbett zurückführen.

Während Martius' medizintheoretische Interessen hauptsächlich von der Frage geleitet wurden, welche Faktoren bei der Krankheitsentstehung zusammenwirkten, ging der Berliner Kliniker Wilhelm His (1863-1934) in seinem Vortrag beim Internistenkongress 1911 insbesondere auf das Problem des Krankheitssitzes ein. ${ }^{23}$ Das Konzept einer Konstitution könne das Problem lösen, dass sich nicht alle Krankheiten nach der Virchowschen Auffassung im

nicht, gehört inhaltlich aber zweifellos zum Diskurs dazu. Jaensch 1933 nennt zwar das Schlagwort, gehört aber inhaltlich nicht zum Diskurs, näheres zu diesem Beitrag siehe unten.

18 Krehl 1911; His 1911; Pfaundler 1911. Zur Bedeutung des Kongresses für die Konstitutionslehre siehe Metzger 2016, 210-211, 213-215.

19 Zu Martius und seinem Beitrag zur Konstitutionslehre siehe Metzger 2016, 209-210, 217219; Krügel 1984.

20 Martius 1923, 21. Siehe auch Martius 1914.

21 Martius 1923, insbes. 20-30, etwa 22: «die alles beherrschende Experimental-Bakteriologie». Die Abgrenzung gegenüber der Bakteriologie wurde in vielen konstitutionspathologischen Veröffentlichungen perpetuiert, etwa Grote 1921, 34.

22 Martius 1923, 30.

23 His 1911. 
Körper klar lokalisieren lassen. Das einengende «Lokalisations-, das Organprinzip, der solidaristisch-anatomische Gedanke» könne mit der Konstitutionslehre überwunden werden. ${ }^{24}$ Auch er stellte die Beobachtung am Krankenbett über lebensfernere Folgerungen anhand Leiche und Laborarbeit.

Trotz einer klinik- und patientenorientierten Rhetorik stellte sich die Moderne Konstitutionslehre in dieser Frühphase streng naturwissenschaftlich auf, auch um dem Vorwurf einer Rückkehr zur überkommenen Humoralpathologie zu begegnen. So imaginierte auch Ludolf Krehl (1861-1937), in diesem Jahr Vorsitzender des Internistenkongresses, in seiner Eröffnungsrede die Erforschung der Konstitution als eine methodische Synthese zwischen naturwissenschaftlich fundierter Grundlagenforschung und reflektierter klinischer Empirie..$^{25}$ Dementsprechend bediente sich die junge Forschungsrichtung in ihrem ersten Jahrzehnt vordringlich der zeitgemäßen Methoden von Vermessung, Statistik, experimenteller Endokrinologie und Vererbungslehre.

Zu Beginn der 1920er Jahre war die Konstitutionsforschung voll etabliert und «Konstitution» fächerübergreifend eines der Schlagworte in medizinischen Veröffentlichungen: Konstitutionsforschung galt nun als «modisch». ${ }^{26}$ Inhaltlich wurde im Laufe der 1920er Jahre die konstitutionspathologische Perspektive immer mehr ausgeweitet und nun auch psychische Faktoren bis hin zur Berücksichtigung der «Gesamtperson» des Kranken eingeschlossen. Dabei wurden insbesondere holistische Anschauungen aufgenommen, die zeitgenössisch überfachlich diskutiert wurden. ${ }^{27}$ «Nur der auf das große Ganze gerichtete Blick kann heute die Medizin vor Einseitigkeiten und Entfremdung schützen», schrieb so auch der konstitutionspathologische Vordenker Theodor Brugsch, der die Ausweitung besonders intensiv vorantrieb und mit dem «Personalismus» eine eigene Fortentwicklung der Konstitutionslehre vertrat. ${ }^{28}$ Auch er kritisierte eine lokalistische Ausrichtung der medizinischen Betrachtungsweise - so sei die «pathologische[n] Anatomie für die Klinik ein Prokrustesbett geworden» - und die damit einhergehende Reduktion der Krankenbetrachtung auf das kranke Organ. Nur die individuelle Betrachtung des Patienten in seiner «psycho-physischen Ganzheit» könne Abhilfe schaffen.

Zentrale Punkte des späteren Krisendiskurses klingen bei Brugsch schon 1920 an: Viele Patienten hätten das Vertrauen in die (Schul-)Medizin verloren und suchten Hilfe bei den «Kurpfuschern» - und daran sei die Medizin

24 His $1911,19$.

25 Krehl 1911.

26 Martius 1923, 105; Bier 1931, 409: «die große Mode».

27 Dazu siehe Metzger 2017, 300. Ein vielzitiertes Beispiel hierfür ist Theodor Brugschs «Personallehre»; Brugsch/Lewy 1926; dazu siehe Gadebusch Bondio 2015, 24-37.

28 Brugsch 1920, 318. 
nicht ganz unschuldig. Die von Brugsch vertretene patientenzentrierte Perspektive solle dieser Entwicklung entgegenwirken. ${ }^{29}$

So wie Brugsch sahen auch andere Autoren in konstitutionspathologischen Ansätzen das Potenzial, «die Medizin» in Forschung und Praxis zu erneuern. Bekannte Vertreter wie Ludolf Krehl oder Louis R. Grote brachten im Kontext der Konstitutionslehre auch noch nach 1920 medizinkritische Gedanken an. ${ }^{30}$ In diesem Sinne kann die Moderne Konstitutionslehre nicht nur als interdisziplinäres Forschungsprogramm oder als Lehrgebäude ihrer Thesen und Inhalte betrachtet werden, sondern auch als eine Erneuerungsbewegung der Medizin.

Ähnliche Fragen nach medizinischem Selbstbild, grundlegenden Denkweisen in Forschung und Praxis sowie nach der zugrundeliegenden Epistemologie wurden auch innerhalb des Krisendiskurses ab der zweiten Hälfte der 1920er Jahre gestellt. ${ }^{31}$ Neben den vieldiskutierten Punkten der wirtschaftlichen Krise der praktizierenden Ärzteschaft, insbesondere in Bezug auf die als übermächtig empfundenen Krankenkassen, der augenscheinlichen Abwanderung vieler Patienten zu Heilpraktikern, den geringen Therapieerfolgen im Vergleich zur Alternativmedizin und einer beschworenen berufsethischen Krise von Ausbildung und Berufsselbstverständnis vieler Kollegen wurde so auch eine wissenschaftliche Krise der Grundlagen der Medizin thematisiert.

Es wurde beklagt, dass die universitären Mediziner zu sehr auf Grundlagenforschung fokussieren, während die Therapie vernachlässigt werde. Zwar verbessere die Forschung die diagnostischen Mittel, doch kaum die Therapiemöglichkeiten. Es bleibe nur, sich entweder wissenschaftlich sauber die weitgehende therapeutische Hilflosigkeit einzugestehen - zum Undank der Patienten - oder auf die traditionellen Therapien der «alten Medizin» zurückzugreifen, wodurch man sich nicht von gewissen alternativmedizinischen Angeboten unterscheide. Schuld an dieser Misere seien die derzeitig dominanten wissenschaftlichen «Grundanschauungen», insbesondere die Zellularpathologie Virchows mit ihrem lokalistischen Krankheitsverständnis und klaren Kausalverknüpfungen sowie der in Forschung und Ausbildung ausgemachte Mechanismus und Reduktionismus, der den wahren Kern des Arztberufes, die menschenzugewandte Bemühung um Linderung von Leiden, empfindlich störe. ${ }^{32}$

29 Brugsch 1920, 318.

30 Krehl 1923, 1-13, vgl. auch 697-706; Grote 1921.

31 Zur «Krise der Medizin» siehe Roelcke 2016; Schmiedebach 2014 und 1989; Hahn 2011, 728-37, 740-50; Geiger 2010; Knipper 2007; Hau 2004; Timmermann 1999; Bothe 1991; Klasen 1984.

32 Bothe 1991, 18-21; Roelcke 2016. Beiträge zur «wissenschaftlichen Krise» sind u.a. Krehl 1926; Sauerbruch 1926; Honigmann 1927; Goldscheider 1927; Gruber 1933. 
Während sich die Beiträge beim Thema wirtschaftlicher Krise weitgehend einig waren, gingen die Meinungen zur wissenschaftlichen Krise auseinander. Manche Autoren erteilten der universitären Forschung eine klare Absage und wandten sich alternativmedizinischen Ansätzen zu, andere bekräftigten das Potenzial der naturwissenschaftlich ausgerichteten Forschung, die die Medizin voranbringen und letztlich aus der vermeintlichen Krise herausführen werde. Zwar neigten die meisten Teilnehmer des Diskurses dazu, den Dienst am Patienten in den Mittelpunkt des medizinischen Schaffens zu rücken, aber die Wege zu einer besseren Medizin wurden verschieden imaginiert. Mal war es der Kampf gegen «Materialismus», sowohl im Sinne von reduktionistischer Betrachtungsweise des Patientenkörpers als auch in Bezug auf monetäres Gewinnstreben, mal die holistische Betrachtung des Patienten in seiner «psycho-physischen Ganzheit», mal die Rückbesinnung auf große Arztgestalten der Vergangenheit und ihre Werte, die als Lösungsvorschläge vorgebracht wurden. ${ }^{33}$

Vor diesem Hintergrund ist es nicht unplausibel anzunehmen, dass auch die Moderne Konstitutionslehre als Lösungsstrategie für die «wissenschaftliche Krise» gehandelt worden sein könnte. Konstitutionspathologische Kritikpunkte an der Gegenwartsmedizin, die seit dem Internistenkongress in Wiesbaden $1911 \mathrm{im}$ Rahmen der Konstitutionslehre immer wieder vorgebracht wurden, kehrten im Krisendiskurs unverändert wieder: Übermäßiger Lokalismus, Reduktionismus und Mechanismus wurden in beiden Diskursen bemängelt, eine patientenferne Labormedizin wurde durchgängig, die Zellularpathologie tendenziell beanstandet. Konstitutionslehre wie Krisendiskurs forderten eine patientenzentrierte Perspektive der Medizin und beriefen sich auf alte klinische Tugenden - selbst wenn die Umsetzung dieser Ideale schwer fiel. ${ }^{34}$

Neben diesen Gemeinsamkeiten gibt es auch inhärente Konflikte zwischen Konstitutionslehre und den Anliegen des Krisendiskurses: Konstitutionslehre forderte intensive Grundlagenforschung; Anwendungsbezug und Therapie waren zunächst völlig nachgeordnet und wurden erst im Laufe der 1920er Jahre zunehmend thematisiert, als der Krisendiskurs schon lief. ${ }^{35}$ Die Methoden der Konstitutionslehre waren nicht nur dezidiert naturwissenschaftlich orientiert, sondern arbeiteten in ihren anthropometrischen und statistischen Spielarten alles andere als auf den Einzelpatienten bezogen. Labormedizin und Tierversuch waren nicht wegzudenken, insbeson-

33 Bothe 1991, 18-21. Vgl. etwa Krehl 1926; Sauerbruch 1926; Honigmann 1927; Goldscheider 1927; Gruber 1933.

34 Metzger 2016, 209-15, 237-38.

35 Metzger 2017, 295-299. 
dere nicht aus dem konstitutionspathologisch bedeutsamen Forschungsfeld der Endokrinologie. Forderungen nach der Betrachtung des Patienten als Individuum verblieben meist im rhetorischen Raum. ${ }^{36}$

Es ist damit zu prüfen, welche Rolle die Konstitutionslehre im Krisendiskurs einnahm. Besaß sie in den Augen der Beiträger zum Krisendiskurs noch ihr ursprüngliches Potenzial für Reform und Erneuerung der Medizin, insbesondere hinsichtlich ihres proklamierten patientenorientierten Blickes? Oder hatte die Konstitutionslehre fünfzehn bis zwanzig Jahre nach ihrer Konzeption bereits enttäuscht und zählte für die Krisenautoren zum kritisierten Establishment? Es ist bezeichnend, dass «Konstitution» im Krisendiskurs weniger in Bezug auf die hier skizzierte «Moderne Konstitutionslehre» thematisiert wurde, als im Zusammenhang mit einer auf humoralpathologischen Prinzipien beruhenden Außenseitermethode, der stark an ihren Begründer geknüpften «Konstitutionstherapie».

\section{Bernhard Aschners «Konstitutionstherapie»}

Der Wiener Gynäkologe Bernhard Aschner veröffentlichte seine Monographie Die Krise der Medizin - Konstitutionstherapie als Ausweg im Jahr 1928, als der Krisendiskurs bereits seit mehreren Jahren geführt wurde. Da im Krisendiskurs besonders beklagt wurde, dass die moderne medizinische Wissenschaft die Therapie vernachlässige, konnte Aschners bereits während des Ersten Weltkrieges entwickeltes Therapiekonzept hier auf besonders fruchtbaren Boden fallen und wurde häufig von krisenbesorgten Ärzten ausgesprochen positiv rezensiert. ${ }^{37}$ Aschners Versuch, mit seiner Konstitutionstherapie langbewährte Verfahren der alten Medizin wiederzubeleben, entsprach zudem der Tendenz vieler Krisenbeiträger, sich orientierungssuchend an ärztliche Lichtgestalten der Vergangenheit zu wenden. Aschner vermochte es außerdem, sich im Krisendiskurs auch durch seine Biographie als unabhängigen kritischen Geist darzustellen, der sich als ehemaliger Experimental- und Laborforscher enttäuscht vom universitär-theoretischen Erkenntnisstreben abgewandt und der wahren Aufgabe eines Arztes zugewandt hatte: dem Heilen.

Bernhard Aschner, geboren 1883, war nach seinem Medizinstudium in Wien zunächst in der experimentellen Hypophysen- und Zwischenhirnforschung tätig; so gelang ihm zu Beginn seiner Laufbahn die erste vollständige Hypophysenentfernung am Hund mit überlebendem Versuchstier. Dann 
wandte er sich der gynäkologischen Hormonforschung zu. Trotz seiner frühen Erfolge hatte er Schwierigkeiten, akademisch Fuß zu fassen: Nach einem ersten gescheiterten Habilitationsversuch in Wien habilitierte er sich 1914 in Halle für Gynäkologie, doch der Erste Weltkrieg, der Tod seines dortigen Förderers und wohl auch seine jüdische Herkunft hemmten eine weitere Karriere. Von 1918 bis zu seiner erzwungenen Emigration 1938 führte er eine sehr erfolgreiche Privatpraxis in Wien und war lediglich als Privatdozent an die dortige Universität angebunden. Akademisch blieb er ein Außenseiter. ${ }^{38}$

Bereits vor oder während des Ersten Weltkrieges wandte sich Aschner humoralpathologischen Therapiemethoden und der «alten Medizin»zu. 1918 prophezeite er dem Aderlass in seiner endokrinologischen Monographie Die Blutdrüsenerkrankungen des Weibes eine große Zukunft. ${ }^{39}$ Die noch junge Moderne Konstitutionslehre interpretierte er als eine Wiederzuwendung zur Humoralpathologie - eine Mindermeinung. 1922 sprach er sich in der Wiener Klinischen Wochenschrift dann klar für eine «moderne $\mathrm{Hu}-$ moralpathologie» aus, die Erkenntnisse der naturwissenschaftlichen Medizin mit den wirksamen Methoden der «alten Medizin» vereinen sollte. «Salinische Abführmittel, Hydrotherapie (insbes. Schwitzkuren) und ganz besonders [...] [der] Aderlaß» seien in der Lage, die weitgehende therapeutische Hilflosigkeit der letzten 100 Jahre zu überwinden. ${ }^{40}$ Eine solche «Renaissance der Humoralpathologie» vermöge es wahrscheinlich, «in absehbarer Zeit» das «Krebsproblem» zu lösen. ${ }^{41}$ Die Anwendung der alten ausleitenden Methoden sei auch der Grund für den Erfolg von «Naturheilärzten und Kurpfuschern».22 Er empfahl die Lektüre von «alten» Größen wie Paracelsus und Hufeland, um vergessenes Heilwissen wieder in den aktiven Therapiegebrauch zu bringen - um dies zu fördern übersetzte er selbst zwischen 1926 und 1932 mehr als 4000 Seiten Paracelsus ins Hochdeutsche, und zwar in einer Weise, die von medizinhistorischen Rezensenten als naiv und anachronistisch bewertet wurde. ${ }^{43}$

Besondere Bedeutung maß Aschner als Frauenarzt jeglicher Form von ausbleibender Menstruation zu, da die dadurch im Körper verbleibenden «schädlichen Stoffwechselprodukte» vielfältige Erkrankungen hervorrufen könnten, zum Beispiel Glaukome, Myome, Dermatosen und Psychosen -

$38 \mathrm{Zu}$ Aschner siehe Brunk-Loch 1995; Geiger 2010, 398-405; Timmermann 1999, 90-93, 2001, 732-737; Klasen 1984, 76-83. Ausführliche Literatur bei Fischer/Voswinckel 2002a. 39 Aschner 1918, 23-24.

40 Aschner 1922, 108.

41 Aschner 1922, 77.

42 Aschner 1922, 108.

43 Dazu siehe Brunk-Loch 1995, 81-97. 
eine typische humoralpathologische Vorstellung. ${ }^{44}$ Sein Ziel ist klar artikuliert: «Ganz besonders möchte ich der Wiedereinführung des Aderlasses auf breitester Basis [...] in der Gesamtmedizin zum Durchbruch verhelfen.» ${ }^{45}$ Diese Grundlagen der «Konstitutionstherapie» sollten sich auch in Aschners späteren Veröffentlichungen nicht ändern, sie wurden lediglich im Detail erweitert und schärfer von der abgelehnten Lokal- und Zellularpathologie abgegrenzt, so auch im Krisen-Buch von 1928.

Nach 1922 widmete sich Aschner damit ganz der Konstitutionstherapie, ihrer Bewerbung und Ausgestaltung. In den 1930er Jahren erprobte er aus seiner Sicht erfolgreich die Anwendung ausleitender Verfahren bei weiblichen Psychiatriepatientinnen, im amerikanischen Exil der Nachkriegszeit beschäftigte er sich mit Konstitutionstherapie bei Rheuma. ${ }^{46}$ Sein Verfahren wurde immer stärker als Außenseitermethode wahrgenommen, so dass es nach 1945 hauptsächlich in alternativmedizinischen Kreisen rezipiert wurde.

Für die 1920er Jahren hingegen und insbesondere den Krisendiskurs ist es schwierig, eine inhaltliche Scheidung von Alternativ- und Schulmedizin zu treffen; ihre Front verlief vielmehr entlang der klaren Linie zwischen approbierten Ärzten und «Kurpfuschern». Aschner selbst wollte als Arzt mit seiner «Renaissance der Humoralpathologie» die universitär gelehrte $\mathrm{Me}$ dizin revolutionieren; er publizierte in medizinischen Fachzeitschriften, sprach vor medizinischen Gesellschaften und trug dort direkte inhaltliche Auseinandersetzungen mit Fachkollegen aus. ${ }^{47}$ Er wurde trotz teils harscher Kritik zwar als Außenseiter, aber nicht als außerhalb der universitären Medizin stehend wahrgenommen. Prinzipiell verschwammen im Krisendiskurs die Grenzen zwischen Methoden der Alternativ- und Schulmedizin; manchen Diskutanten schien es in der Krise sehr ungewiss zu sein, in welche

44 Etwa Aschner 1924, 1279. Eindrücklich Aschner 1933, 15: «L. Adler bemerkte, ob ich denn wirklich glaube, daß das 'giftige' Menstrualblut die Psychosen auslöse, so möchte ich erwidern, daß das Menstrualblut tatsächlich giftig ist, aber nicht so sehr für andere Personen, wie der alte Volksglauben lautet [...], sondern für die betreffende Trägerin selbst, nämlich dann, wenn diese Stoffwechselprodukte nicht genügend ausgeschieden werden, sondern im Körper verbleiben. [...] Tatsächlich fühlen sich auch die Frauen nach diesem monatlichen Ausleerungsvorgang im ganzen Körper gereinigt und befreit.»

45 Aschner 1922, 109.

46 Aschner 1933: Heilerfolge der Konstitutionstherapie bei weiblichen Geisteskranken; zu seinen Rheuma-Studien siehe Brunk-Loch 1995, 67-75.

47 Etwa in der Wiener Medizinischen Wochenschrift als schriftlicher Disput (Borak 1926 in Antwort auf einen Artikel Aschners, mit Erwiderung Aschners) oder in der Gesellschaft der Ärzte in Wien: Vortrag eines Mitglieds zu «Sektentum in der Medizin» mit Erwiderung Aschners (Gesellschaft der Ärzte in Wien 1931a). Nach dieser öffentlichen Auseinandersetzung wurde Aschner aufgefordert, in der nächsten Sitzung vom 12. Juni 1931 seine Position zu verteidigen; er stellte dort eine durch Konstitutionstherapie geheilte Patientin vor, anschließende kontroverse Diskussion (Gesellschaft der Ärzte in Wien 1931b). 
Richtung sich die Medizin zukünftig entwickeln würde. ${ }^{48}$ In dieser Stimmung konnte es Aschner nicht unmöglich erscheinen, dass seine Prognose einer «Renaissance der Humoralpathologie» wahr werden könnte.

Denkbar fern steht Aschners Konstitutionstherapie, wie er sie 1928 als «Ausweg aus der Krise der Medizin» anbot, der Modernen Konstitutionslehre. Zwar vereinte die Konstitutionslehre der 1920er Jahre sehr heterogene Ansätze und Lehrmeinungen, aber Aschner hatte sich zum Zeitpunkt des Krisendiskurses bereits inhaltlich wie rezeptorisch so sehr von dieser entfernt, dass man seine Konstitutionstherapie nicht unter die Moderne Konstitutionslehre subsumieren kann, sondern als eigenständigen Ansatz betrachten muss. Diese Unterscheidung ist insofern bedeutsam, als dass Aschner sowohl von Klasen als auch von Timmermann als Hauptzeuge für die These aufgeführt wird, die Konstitutionslehre sei in der Diskussion um die «Krise der Medizin» prominent als Lösungsvorschlag verhandelt worden. ${ }^{49}$

Die von Aschner propagierte Konstitutionstherapie lässt sich bereits inhaltlich in entscheidenden Punkten nicht mit der Modernen Konstitutionslehre vereinen: Bereits sein Konstitutionskonzept schloß Aschner an das der vormodernen Humoralpathologie an; sie sei eine umfassende, sich nach «Lebensalter und Geschlechtsphasen» ändernde Körperverfassung ${ }^{50}$ und durch ableitende Verfahren beeinflussbar: In der praktischen Anwendung habe sich dieser «Konstitutionsbegriff in seiner erweiterten Fassung seit Jahrtausenden bewährt und wird sich bei richtiger Anwendung auch weiterhin bewähren». ${ }^{51}$ Damit ging Aschner bereits 1924 sehr viel weiter als die meisten Konstitutionspathologen seiner Zeit, die nicht selten «Konstitution» nur für den durch Vererbung festgelegten oder angeborenen Körperzustand anwandten. ${ }^{52}$ Die Beschäftigung mit Vererbung - ein für die überwiegenden Anzahl an Konstitutionsforschern sehr wichtiges Thema - wies Aschner als irrelevant zurück: Konstitution war für ihn immer durch Lebensweise und

48 Vgl. Bier zur Homöopathie, Bothe 1991, 18-19.

49 Siehe oben unter «Forschungsstand und Vorgehen».

50 Aschner 1924, 174.

51 Aschner 1924, 175. Ebd. 173-75 widmet Aschner mehrere Seiten der Definition, insbesondere in Auseinandersetzung mit konstitutionspathologischen Autoren. Der Text liest sich häufig widersprüchlich, die Tendenz zur Humoralpathologie ist hingegen klar: «Nur die geläuterte Wiederanwendung des Konstitutionsbegriffes im Sinne der alten klassischen Medizin [...] hat Aussicht auf praktische Brauchbarkeit», Aschner 1924, 179. Aschner 1928, 35-43, konzentriert sich auf die Definition der Konstitutionstherapie, in deren Dienst der Konstitutionsbegriff gestellt wird. Konstitutionstherapie sei die «therapeutische Erfassung des Gesamtorganismus mit besonderer Berücksichtigung seiner humoralen Eigenschaften», ebd. 43. - Siehe auch die diesbezügliche Kritik des Internisten Paul Saxl nach Aschners Vortrag am 12. Juni 1931 vor der Gesellschaft der Ärzte in Wien, nämlich dass Aschner, «wenn er von Konstitution spricht, etwas anderes meint, als man sonst unter Konstitution versteht». Gesellschaft der Ärzte in Wien 1931b, 923.

52 Dazu siehe Metzger 2017, 291-295. 
Therapie beeinflussbar, die Frage nach ihrer Ursache nutzlos. Zwar erkannten auch konventionelle Konstitutionspathologen Umwelt- und andere äuBere Faktoren als Einflüsse auf die individuelle Konstitution an und machten gegen Ende der 1920er Jahre vermehrt therapeutische Vorschläge zur Verbesserung der Konstitution, ${ }^{53}$ aber Aschners stark fluides Konzept der Konstitution stach hervor - es war ganz im humoralpathologischen Körperbild verhaftet. Gleichzeitig entsprach nichts aus dem Herzstück Aschners Theorie - die Behandlungsmethoden - in irgendeiner Form dem, was zur gleichen Zeit in der modernen Konstitutionslehre verhandelt wurde.

Aschner setzte sich außerdem kaum mit den Themen und Diskussionen der Konstitutionslehre der 1920er Jahre auseinander. Er rezipierte Schriften bekannter Konstitutionspathologen nur oberflächlich und lediglich bis etwa 1921 (Kretschmer). Noch 1928 zeichnete er in seiner Krisen-Monographie das Bild einer Konstitutionslehre, die sich hauptsächlich für Körpertypologien interessiere, dabei hatten sich die meisten von ihm zitierten Autoren zu diesem Zeitpunkt bereits von der Typologisierung verabschiedet. ${ }^{54}$

Auch selbst grenzte sich Aschner zunehmend von der Konstitutionslehre ab. Während er 1918 und 1922 seine Schriften noch in der «jetzt zu einer eigenen Wissenschaft gewordenen $»^{55}$ Konstitutionslehre verortete und gar seinen Aufsatz in der Wiener Klinischen Wochenschrift 1922 mit dem Versprechen beginnt, in diesem «die Konstitutionslehre in einer Beleuchtung zu zeigen, welche vielleicht geeignet ist, sie [...] für den allgemeinen ärztlichen Gebrauch zugänglich zu machen ${ }^{56}$, distanziert er sich bald von eben dieser Konstitutionslehre. Noch im selben Aufsatz schließt er:

«Die Lehre von der inneren Sekretion und von der Konstitution sind nur Etappen auf dem Wege zu einer vorwiegend, wenn auch nicht ausschließlich humoral begründeten und daher universalistischeren medizinischen Auffassung des Krankheitsgeschehens..$^{57}$

Schärfer formulierte Aschner diese Fortentwicklung bzw. Überwindung der defizitären zeitgenössischen Konstitutionspathologie in den Werken ab 1924: Die moderne Medizin mit den «Grundanschauungen» von Lokal- und Zellu-

53 Metzger 2017, 295-299.

54 Besonders augenfällig ist dies bei Theodor Brugsch, der bei Aschner als Proponent eines einseitigen Typensystems dargestellt wird. Aschner bezieht sich dabei auf Brugschs Arbeiten vor 1918 - bereits wenige Jahre später hatte sich Brugsch davon distanziert und verfolgte seit 1922 sein individualistisches Konzept eines «Personalismus». Von Aschners 1928 genannten Autoren verfolgte nur noch Ernst Kretschmer seine Typenlehre (und dies bis in die Bundesrepublik). Dazu siehe Metzger 2017, insbes. 283 (Kretschmer), 286 (Brugsch) u. 290 (Bauer).

55 Aschner 1918, 19.

56 Aschner 1922, 73.

57 Aschner 1922, 109. Aschners Begriff von «humoral» ist humoralpathologisch und anders als bei manchen Zeitgenossen nicht im übertragenen Sinne auf die Endokrinologie bezogen. 
larpathologie und ihrem naturwissenschaftlichen Kausalgedanken habe versagt; auch «die meisten Konstitutionsforscher» seien noch in diesem Denken verhaftet, sie stünden «noch durchaus auf dem Boden der Morphologie, der Vererbungslehre und der Zellularpathologie». Die Konstitutionslehre sei «bezeichnenderweise auch therapeutisch vollkommen unfruchtbar geblieben». ${ }^{58}$ Man stehe vor einem «Systemwechsel in der gesamten Medizin» ${ }^{59}$, die $\mathrm{Zu}$ kunft gehöre einer «geläuterten Humoralpathologie». ${ }^{60}$

Während Aschners Konzept im Krisendiskurs durch niedergelassene Ärzte und in nicht-medizinischen Publikationsorganen meist positiv rezipiert wurde, lehnten die universitären Rezensenten die Konstitutionstherapie fast durchgängig ab. ${ }^{61}$ «Vollständige Fassungslosigkeit» musste ein Wiener Kollege bekennen, «wenn man in Kenntnis der Tatsachen seines Faches» Aschners Therapievorschläge läse, ${ }^{62}$ und der Konstitutionspathologe Julius Bauer fragte:

«Hat dieses sonderbare Buch wirklich derselbe Bernhard Aschner geschrieben, derselbe verdienstvolle Forscher, dem wir grundlegende Untersuchungen auf dem Gebiete der inneren Sekretion, insbesondere der Hypophysenpathologie und -physiologie verdanken?» ${ }^{63}$

Besondere Kritik schlug Aschners Argumentationsweise entgegen, die entweder auf Einzelfallbeispiele aufbaute oder auf die jahrhundertelange Erprobung durch die «alte Medizin» verwies: «Keinerlei Beweiskraft» komme Aschners Fallbeispielen zu, urteilte derselbe Julius Bauer nach einem Vortrag Aschners vor der Gesellschaft der Ärzte in Wien am 12. Juni 1931, denn «wenn der Vortr[agende] alle seine Fälle [...] mitteilen wird, wird man sicherlich auch von genug Fällen erfahren, die vom Vortr[agenden] nicht geheilt wurden». Ähnlich äußerte sich der Psychiater Heinrich von Kogerer zum selben Vortrag: «Nur eine große Zahl von ähnlichen Verläufen [...] könnte zum Schluß berechtigen, daß keine Spontanremission vorliegt», ein anderer Hörer kritisiert Aschners «Verallgemeinerungstendenz aus vereinzelten Beobachtungen». ${ }^{64}$ Als Antwort auf solche Vorwürfe baute Aschner seine Monographie Heilerfolge der Konstitutionstherapie bei weiblichen Geisteskranken (1933) auf Fallschilderungen von hunderten von erfolgreichen und einem Dutzend missglückter Therapien

58 Aschner 1928, 26.

59 Aschner 1928, 34.

60 Aschner 1928, 28.

61 Brunk-Loch 1995, 77-80. Siehe Timmermann 2001, 734, für die harsche Kritik durch die Konstitutionsforscher Julius Bauer und Wilhelm His. Positive Rezensionen durch nicht-universitäre Partizipienten am Krisendiskurs: Lehfeldt 1927; U.T. 1928; Moses 1929; Schmidt 1929.

62 Borak 1926, 405.

63 Bauer 1924.

64 Gesellschaft der Ärzte in Wien 1931b, 923. 
auf. Dieser Ausflug in die Psychiatrie wird in den Rezensionen von Fachkollegen als methodisch «reichlich unkritisch» bis «dilettantisch» abgetan. Aschners Duktus, der Fallbeschreibungen Heilwunderberichten ähnlich machte, kam bei wissenschaftlich arbeitenden Kollegen nicht gut an und trennte sein Werk auch methodisch grundlegend von den Arbeiten führender Konstitutionspathologen wie Julius Bauer oder Theodor Brugsch.

\section{Erwähnungen von Konstitution im Krisendiskurs}

Außerhalb von Aschners Konstitutionstherapie wurde das Stichwort «Konstitution» nur wenig im Krisendiskurs erwähnt, entsprechendes gilt für die Moderne Konstitutionslehre als zeitgenössischeForschungsrichtung oder Anschauungsweise ${ }^{65}$ So nennt etwa Erwin Liek in seinem für den Krisendiskurs zentralen Buch Der Arzt und seine Sendung (1926) das Stichwort nur beiläufig, als er das Verschwinden des klassischen Hausarztes lamentiert, der als «ärztliche Freund und Berater [...] der berufenste Sachverständige in Fragen der Konstitution und des Erbgangs» gewesen sei. Die zeitgenössische Konstitutions- und Vererbungsforschung versuche nur die Lücke zu füllen, die der «leider ausgestorbene Hausarzt» gerissen habe. ${ }^{66}$ Nun interessierte sich Liek vielleicht als Chirurg nicht besonders für die von den inneren Fächern geprägte Konstitutionslehre, zumal sein Schwerpunkt im Krisendiskurs auf der praktischen Tätigkeit der Ärzte lag, nicht auf theoretischen Diskussionen über das richtige wissenschaftliche Paradigma. ${ }^{67}$ Doch zeigt sein Beispiel gut, wie nebensächlich und vereinzelt Erwähnungen von «Konstitution» häufig in die Beiträge zum Krisendiskurs eingebettet sind.

So verwiesen auch andere krisenbesorgte Ärzte kurz und knapp auf «Konstitution», wenn sie bedeutungsvolle Themen innerhalb der zeitgenössischen Medizin zur Sprache brachten. «Der alte Begriff der Konstitution ist wieder hochaktuell», schrieb der Internist Wilhelm His 1930 in einem Überblicksartikel zur «Krise in der Medizin» in der Illustrierten Die Woche. ${ }^{68}$ Man solle weiter zum «Ganzen des Organismus» inklusive der psychischen

65 Krehl 1926; Sauerbruch 1926; Liek 1926; Goldscheider 1927; Honigmann 1927; Honigmann 1928a; Brugsch 1928; Diepgen 1928; Grote 1928; Bumke 1929; Huismans 1929; Sigerist 1930; His 1930; Much 1931; Bier 1931; Gruber 1933.

66 Liek 1926, 99. «Er sah durch Geschlechter hindurch die Kinder geboren werden und aufwachsen, sah ihre Reaktion auf Krankheitskeime, lernte ihre Stärken und Schwächen genau kennen. Ich glaube nicht, daß die heutige wissenschaftliche Forschung jetzt schon das ersetzen kann, was die deutsche Familie an ihrem Hausarzt verloren hat.»

67 Zu Liek siehe Schmiedebach 1989; Jehs 1994; Timmermann 1999, 69-77; Geiger 2010, 388393.

68 His 1930, 790 (Wiederabdruck: His 1932). 
Ebene forschen. Sein emeritierter Berliner Kollege Alfred Goldscheider zählte in der Deutschen Medizinischen Wochenschrift die individuelle Konstitution als einen von (vielen) Punkten auf, die man als guter praktischer Arzt berücksichtigen sollte. ${ }^{69}$

Längere Diskussionen der Konstitutionslehre, in denen die Autoren eine dezidierte positive oder negative Haltung gegenüber dieser ausdrücken, sind im Krisendiskurs selten. Heraus sticht Hans Much, Professor für Innere Medizin in Hamburg, der der Konstitutionslehre äußerst positiv gegenüberstand. Much wurde (und wird) als wichtiger Impulsgeber für den Krisendiskurs wahrgenommen, obwohl er das Stichwort der «Krise» fast nie aussprach, also sich nicht selbst ausdrücklich dem Diskurs zuordnete. ${ }^{70}$ Sein Werkverzeichnis nennt keine einzige Schrift, die «Krise» im Titel trägt. ${ }^{71}$

Much war sowohl als medizinisch-essayistischer als auch schöngeistiger Schriftsteller äußerst produktiv und kam immer wieder auf die seiner Meinung nach zentrale Rolle der Konstitution für medizinische Forschung und Praxis zu sprechen, zu der er auch selbst forsche. So etwa in seinen vielgelesenen Büchern Hippokrates der Große (1926) und Das Wesen der Heilkunde (1928), die das Stichwort «Krise» beide nicht nennen. Allerdings setzte er sich im Juni 1930 zumindest thematisch in einem Vortrag auf Einladung der Freien Studentenschaft Freiburg mit dem Zustand der medizinischen Wissenschaft auseinander, wobei er die «Krise» zumindest kurz erwähnte. ${ }^{72}$ Der mit einem überschäumenden Ego ausgestattete Much versuchte sich lieber an eigenen Begriffsprägungen: So fragte er im Titel des Vortrags «Steht die scholastische Medizin vor einem Bankerott?» Seine Antwort: Ja, sofern sie weiterhin Leben als etwas Statisches begreife, anstatt der $«$ Dynamis des Lebendigen ${ }^{73}$ gerecht zu werden, was man am besten durch eine echt individuumsbezogene, den Menschen in seiner Ganzheit betrachtende Konstitutionslehre erreichen könne: «Die Gesamtkonstitution zu erfassen ist das einzige Ziel einer dynamisch gerichteten lebendigen Medizin.» ${ }^{74}$ Als «zusammenfassendes, überblickendes Fach» sei nur die

69 Goldscheider 1927, 377. Zu Goldscheiders Artikelserie zur Krise in der DMW siehe auch Hau 2004, 116-117.

70 Sekundärliteratur zu Much im Krisendiskurs: Bothe 1991, 36-37; Timmermann 1999, 83 89. Biographisches: Wirtz 1991; Schulze-Rath 1993; weitere Literatur bei Timmermann 1999,84 n. 65.

71 Wirtz 1991, 123-147.

72 Much 1931, 3: Er lasse die «berechtigten Kritiken» der Krisendiskutanten beiseite und konzentriere sich im Folgenden auf die Zukunft; denn es könne «nichts mehr nützen, immer blos auf die vorhandenen Schäden hinzuweisen. Man muß neue Wege zu sehen und zu gehen versuchen».

73 Much 1931, 15.

74 Much 1931, 33. 
Konstitutionslehre (oder «individuelle Bio-Pathologie») dazu in der Lage, die versteifte «Scholastik» und das «löcherige System» zu überwinden. ${ }^{75}$ Zur Weiterentwicklung der Konstitutionslehre brachte Much dementsprechend auch ausführliche konzeptuelle Vorschläge ein, z.B. ein abgestuftes System verschiedener Konstitutionsgrade. ${ }^{76}$

Häufig wurden die Schriften Muchs im Krisendiskurs aufgegriffen. So zitierte etwa der Kölner Klinikchefarzt Laurenz Huismans in seinem Beitrag zur Krise, der in der katholischen Zeitschrift Hochland erschien, aus Muchs Monographie Hippokrates der Große: «Auf die Konstitution Einfluß zu gewinnen, ist das $\mathrm{A}$ und $\mathrm{O}$ der Medizin. ${ }^{77}$ Vollmundig verkündete Huismans dort «Die Konstitution ist wieder einmal Trumpf», um seinem nicht-medizinischen Publikum die Anliegen der Gegenwartsmedizin zu beschreiben.

Der Berliner Ordinarius August Bier, wie Liek Chirurg, ist im Krisendiskurs hauptsächlich für seine positive Haltung gegenüber der Homöopathie bekannt, übte aber auch starke Kritik an der Konstitutionslehre. In einer historisierenden Arbeit zum «Hippokratismus», die lediglich auf den Krisendiskurs anspielt, beklagte er die Auswüchse der modernen Konstitutionslehre und warf ihr «echt moderne[r] Uebertreibung und Einseitigkeit» vor. ${ }^{78}$ Gerade weil er sich selbst auf diesem Feld aus- und die Wichtigkeit des Themas anerkenne, könne er die «ungenießbare» Konstitutionslehre realistisch einschätzen: Was ihr fehle, sei der übergeordnete Begriff einer «generellen Physis», ohne den «die ganze Konstitution in der Luft schwebt». ${ }^{79} \mathrm{Im}$ Rückgriff auf dieses «hippokratische» Konzept sah Bier das Potenzial für die Schulmedizin der Zukunft - die Krise erwähnte er an dieser Stelle allerdings mit keinem Wort. ${ }^{80}$

Während Much und Bier die erwähnten Beiträge nicht explizit dem Krisendiskurs zuordneten, nutzte Theodor Brugsch einen Vortrag, um im Rahmen der Krisendiskussion starke Kritik an der Konstitutionslehre anzubringen: Im Wintersemester 1927/28 war er von Henry E. Sigerist nach Leipzig eingeladen worden, um in einer Vortragsreihe «Grundlagen und Ziele der Medizin der Gegenwart» zu sprechen, die explizit dem Krisendiskurs ge-

75 Much 1931, 29-30.

76 Etwa Much 1928, 61 und 137.

77 Huismans 1929, 467. Zu Huismans siehe Klasen 1984, 158. Das Much-Zitat steht bei Much 1926, 67. Die Idee, «auf die Konstitution Einfluß zu gewinnen» wird innerhalb der Konstitutionslehre gegen Ende der 1920er Jahre hin wichtiger, siehe Metzger 2017, 295-299.

78 Bier 1931, 409. Zu Bier im Krisendiskurs siehe Doms 2005, insbes. 270-278; Timmermann 1999, 77-83; Lammel 1998, 181-98; Bothe 1991, 17-19. Zur Person: Lammel 1994; Fischer/ Voswinckel 2002b mit weiterführender Literatur.

79 Bier 1931, 409-10. Zu Biers Physis-Begriff mit kritischer Einordnung siehe Lammel 1998, $186-90,94-95$.

80 Bier 1931, 410. 
widmet war. In seinem Beitrag «Die Klinik» sprach Brugsch sich für die holistische Betrachtung des «Menschen in vollem Umfange» aus, wie er es in seiner Fortentwicklung der konstitutionellen Idee, dem «Personalismus» fordere. ${ }^{81}$ Die herkömmlichen Konstitutionsforscher reduzierten den Patienten auf körperliche, auch ererbte Eigenschaften und dächten deshalb immer noch zu traditionell innerhalb der «abstrakten Krankheitslehre». ${ }^{82}$

Neben diesen wenigen gegenwartsbezogenen Auseinandersetzungen wurde die Konstitutionslehre im Krisendiskurs gerne als Phänomen einer historischen Entwicklung diskutiert. Dabei versuchten die jeweiligen Autoren eine historische Herleitung der in der Gegenwart wahrgenommenen Probleme der «wissenschaftlichen Krise». Alle Autoren dieser Gruppe führten zunächst aus, in welche angebliche Sackgassen sich die naturwissenschaftlich orientierte Medizin des 19. Jahrhunderts manövriert habe, bevor die gegenläufigen, aktuelleren Entwicklungen, darunter etwa die Konstitutionslehre, dargestellt und ggf. bewertet wurden. Repräsentativ für diese historisierende Einbettung von Krise und Konstitutionslehre ist etwa Paul Diepgens Artikel in der $D M W$ zur Geschichte der «gegenwärtigen Krise»: 83 Als Medizinhistoriker sah Diepgen die Konstitutionslehre zusammen mit dem Ganzheits-Gedanken als einen der bedeutsamsten Umschwünge in der jüngeren Medizin und zählte sie unter die «modernsten Auffassungen der Medizin». ${ }^{84}$ In dieser wie auch anderen medizinhistorischen Arbeiten zum Thema ${ }^{85}$ stellte er die Konstitutionslehre nicht nur als Gegenentwurf zur überkommenen Zellularpathologie dar, sondern scheint ihr wie dieser den Status einer «grundlegenden Betrachtungsweise», eines Paradigmas, zuzuweisen. ${ }^{86}$ Ähnlich wie Diepgen betteten auch Oswald Bumke, Georg Benno Gruber und der bereits erwähnte Alfred Goldscheider die Konstitutionslehre weitgehend neutral in einen historischen Abriss ein. ${ }^{87}$

Drei Beiträge zum Krisendiskurs hingegen stellten in ihren historischen Herleitungen zwischen Konstitutionslehre und «Krise» einen engeren $\mathrm{Zu}$ -

81 Brusch 1928, 67.

82 Brugsch 1928, 70-71.

83 Diepgen 1928.

84 Diepgen 1928, 2174.

85 Diepgen 1926; 1928a; 1933; 1934.

86 Diepgen 1928. Vgl. auch Diepgen 1955, dessen letzter Band unter der Überschrift «Die Heilkunde vom Beginn der Zellularpathologie bis zu den Anfängen der modernen Konstitutionslehre» steht und zu großen Teilen zuvor Publiziertes verwertet. Ebd. S. 293: «Die aus der praktischen Erfahrung entstandene und durch den Energetismus theoretisch gestützte Konstitutionspathologie und erst recht das neu erwachte Interesse für die Seele des kranken Menschen gingen in derselben Richtung. So entstand [...] als Leitidee der Medizin des 20. Jahrhunderts des Ruf nach Synthese, Ganzheitsbetrachtung und funktionellem Denken. Es war ein Triumph der Kraft über den Stoff.»

87 Goldscheider 1927, 290 und 376; Gruber 1933, 804; Bumke 1929, 9; Diepgen 1928, 2173. 
sammenhang her. Für Ferdinand Sauerbruch und Henry E. Sigerist war die Konstitutionslehre Ausdruck eines Umschwungspunktes in der Medizin entsprechend der ursprünglichen medizinischen Bedeutung des Begriffs «Krise». Sauerbruchs stark rezipierter Vortrag und Artikel in Die Naturwissenschaften von 1926 sah die «Krise» als Wende hin zu «diese[r] neue[n] Zeit, die kommen muss und kommen wird»:

«Die Umstellung hat schon begonnen. Überall legt man wieder mehr Wert auf allgemeine Bedingungen, die in der Persönlichkeit des Kranken liegen. Konstitutionspathologie, Vererbungslehre, Einschätzung von Luft, Licht und Klima als Heilfaktoren sind weitere Zeichen dieses Wandels.» ${ }^{88}$

Sigerist argumentierte 1930 ähnlich in den Neuen Blättern für den Sozialismus, die Krise der Medizin sei ein Wendepunkt hin zu einem neuen medizinischen Menschenbild. Im historischen Verlauf hätten Konstitutionslehre sowie Freud und andere tiefenpsychologische Ansätze die entscheidenden Perspektivänderungen dazu beigesteuert. ${ }^{89}$ Für beide Autoren musste der Wandel nicht aktiv betrieben werden; die Konstitutionslehre war vielmehr eher ein Symptom dieser historischen Entwicklung, die in Eigengesetzlichkeit verlief.

$\mathrm{Zu}$ einer ganz anderen Einschätzung der historischen Rolle der Konstitutionslehre kam Ludolf Krehl, der immerhin ab 1911 zu den Protagonisten der damals noch jungen Konstitutionslehre gehört hatte: In einer seiner wenigen Äußerungen zu aktuellen Fragen der Medizin in der Münchener Medizinischen Wochenschrift stellte er die These auf, die «Krise» sei mit den Themen der Konstitutionslehre (und anderen) kausal verknüpft: Sie sei entstanden durch den Widerspruch zwischen physiologisch-naturwissenschaftlichen Forschungsmethoden und den sich aufdrängenden «alten» Problemen von Konstitution und Vererbung, aber auch diversen neuen Problemen (psychologische Fragen, Immunität, Individualität, Person). ${ }^{90}$

Krehls Einschätzung wurde auch durch den Gießener Internisten und Medizinhistoriker Georg Honigmann weitergetragen, der zu den zentralen Gestalten des Krisendiskurses wie auch der daraus hervorgegangenen Gründung der Zeitschrift Hippokrates gehörte. ${ }^{91}$ Honigmanns viel wahrgenommene Beiträge zum Krisendiskurs argumentierten mit der historischen Abfolge medizinischer Systeme bis hin zur einseitig naturwissenschaftlich ausgerichteten Zellularpathologie, denen er den einzig zulässigen Grund- und Zielgedanken der Medizin

88 Alle Zitate Sauerbruch 1926, 1089.

89 Sigerist 1930. Sigerist war Mitherausgeber der Neuen Blätter für den Sozialismus, Jaehn $1991,45$.

90 Krehl 1926, 1548.

91 Honigmann 1927, 1262. Zu Honigmann siehe Knipper 2007, 369-74, 381-94. Zur Zeitschrift siehe Bothe 1991. 
gegenüberstellte, Menschen zu heilen. ${ }^{92}$ Die Moderne Konstitutionslehre nannte er als Beispiel für eine neue «Gedankenrichtung» ungleich «unserer mechanistisch-generalisierenden Methodik», ${ }^{93}$ die aus dem aktuellen Bedürfnis nach einer Betrachtung des ganzen Menschen in seiner individuellen «Einheit und Einzelart» entstanden sei. ${ }^{94}$ Es brauche allerdings noch viel Forschung, um die therapeutischen Herausforderungen dieser «Grundanschauung» zu lösen..$^{95}$ Vor allem aber müsse die epistemische Krise überwunden werden, die sich durch den Konflikt zwischen den neuartigen klinisch orientierten, «ärztlichen» Betrachtungsweisen und dem «naturwissenschaftliche[n] Gewissen» der Zellularpathologie ergeben habe - die These Krehls..$^{96}$ Ähnlich äußerte sich auch der medizintheoretisch interessierte Internist Louis R. Grote. ${ }^{97}$

Ganz abseits von den genannten historisierend argumentierenden Autoren steht der eher randständige Konstitutionsforscher Walther Jaensch, der 1933 recht spät in einem Beitrag in der $D M W$ versuchte, ähnlich wie Aschner sein eigenes Diagnostik- und Therapiekonzept durch die Assoziation mit dem Krisendiskurs zu bewerben. ${ }^{98}$ Obwohl Jaensch den Aufsatz durch den Titel «Konstitutionsmedizin und Kulturprobleme in der Krise der Gegenwart» und den ersten Satz explizit an den Krisendiskurs anband, sprach er dann de facto über etwas ganz anderes, nämlich wie im neuen nationalsozialistischen Wertegefüge seine spezielle Spielart der Konstitutionslehre und besonders auch sein privates «Ambulatorium für Konstitutionsmedizin» Rasse und Volk zu Diensten sein könnte. Es handelte sich dabei nicht um einen Lösungsvorschlag für die «Krise der Medizin». Jaensch begrüßte die «allgemeinen Umwälzungen der Gegenwart» mit Verve und scheint damit die «Krise der Medizin» als erledigt zu betrachten. Die Hinwendung zur Ganzheit, die sich im Krisendiskurs entwickelte, sei nun vollendet worden in der «restlosen Unterstellung» des Individuums unter die Ganzheit des Staates; die Leib-SeeleFrage wurde durch die «unlösliche Einheit blutmäßiger Gebundenheit» beantwortet. ${ }^{99}$ Jaenschs Beitrag verdeutlicht damit auch, wie der Krisendiskurs

92 Etwa Honigmann 1927; 1928. Ebenso bereits Honigmann 1924.

93 Honigmann 1928a, 14.

94 Honigmann 1927, 1216 und 1263.

95 Honigmann 1927, 1376-77.

96 Honigmann 1927, 1262.

97 Grote 1928.

98 Jaensch 1933. Walther Jaensch entwickelte während der Weimarer Jahre eine Methode der Kapillarmikroskopie zur Diagnose mangelnder körperlicher und seelischer Entwicklung, die in der Konstitutionslehre zunächst wenig ernstgenommen wurde. Während des NS konnte er es durch geschicktes politisches Navigieren zu einigem Erfolg bringen. Hau 2015; Kölch 2006, 242-258.

99 Jaensch 1933, 1089. 
ab 1933 unter gänzlich veränderten Vorzeichen fortgesetzt wurde, weshalb eine Beschränkung der Untersuchungszeit bis 1933 naheliegt.

\section{Personelle Verschränkungen}

Bei den bis hierhin diskutierten Autoren fällt auf, dass sich mehrere sowohl im Konstitutions- als auch im Krisendiskurs zu Zukunftsfragen der Medizin äußerten. So waren Ludolf Krehl und Wilhelm His 1911 maßgeblich an der Initiierung der modernen Konstitutionslehre und der Erstellung ihres Forschungsprogrammes beteiligt gewesen. ${ }^{100}$ Theodor Brugsch war zunächst als Konstitutionspathologe aufgetreten, bevor er mit dem «Personalismus» seine eigene Weiterentwicklung einer auf das Individuum bezogengen Medizin verfolgte. Weniger prominent publizierten Hans Much und Walter Jaensch zu Konstitutionsthemen, allerdings dies auch während der Jahre des Krisendiskurses. Alfred Goldscheider und August Bier bezeichneten sich selbst als in die Konstitutionslehre involviert bzw. interessiert, veröffentlichten dazu aber kaum. Friedrich Kraus und Louis R. Grote, beide als Konstitutionspathologen bekannt, äußerten sich zwar im Krisendiskurs, gingen dort aber nicht oder kaum (Grote) auf Konstitutionslehre ein. ${ }^{101}$ Außer Bier waren alle der genannten Autoren Internisten, also Vertreter des Faches, das sich in den 1910er und 20er Jahren der Konstitutionslehre besonders widmete. ${ }^{102}$

Bei den meisten der Genannten lässt sich beobachten, dass die zeitgenössische Konstitutionslehre in ihren Beiträgen zum Krisendiskurs nur eine geringe Rolle spielte. Außer von Much wird die Konstitutionslehre entweder wie von Bier und Brugsch abgelehnt, nur kurz auf sie verwiesen, ganz aus der Krisendiskussion herausgehalten oder diese als Teil von historischen Abrissen erwähnt. Ein Grund dafür ist sicherlich, dass die Prominentesten der Genannten - Ludolf Krehl, Wilhelm His, Theodor Brugsch und Friedrich Kraus - zwar konstitutiv an der Anfangsphase der Konstitutionslehre beteiligt waren, sich ihre inhaltlichen Schwerpunkte allerdings bis zum Krisendiskurs in andere Richtungen entwickelt hatten. ${ }^{103}$

100 Metzger 2016, 210, 213-15 u. 230-32.

101 Kraus 1931; Kraus 1932; Grote 1930a. Grote 1928, 49, nennt kurz die Konstitutionslehre als gescheitertes Beispiel für die Unmöglichkeit, naturwissenschaftliche Medizin und individualisierende klinische Betrachtung in einem wissenschaftlichen System zu vereinen. $\mathrm{Zu}$ den Beiträgen von Kraus und Grote zur Konstitutionslehre siehe Metzger 2016, 216-17 und 222 (Kraus) sowie 228-30 (Grote).

102 Metzger 2017, 273.

103 Friedrich Kraus stieß mit seiner Syzygiologie und Personenlehre in philosophische Gefilde vor, während Ludolf Krehl sich mit Psychosomatik und therapeutischen Fragen auseinandersetzte. 
Das gemeinsame Moment für die personellen Überschneidungen zwischen Krisendiskurs und Konstitutionslehre scheint mithin weniger die inhaltliche Verknüpfung zwischen den beiden Diskursfeldern zu sein, wenngleich diese auch bestand. Vielmehr fühlten sich diese Autoren dazu berufen, ihre Meinung zu den großen Richtungsfragen der Medizin zu äußern, sowohl unter dem Dach der Konstitutionslehre, als auch im Krisendiskurs. Standen die frühen Beiträge von Krehl, Kraus, His und Goldscheider zur Konstitutionslehre noch unter dem Vorzeichen einer Aufbruchsstimmung, so äußerten sie sich im Krisendiskurs als eminente Größen der Inneren Medizin: Alle vier waren zu diesem Zeitpunkt bereits emeritiert oder standen kurz vor der Emeritierung. ${ }^{104}$ Umgekehrt nutzten der etwa fünfundzwanzig Jahre jüngere Louis R. Grote (Jg. 1886, habilitiert 1918) und der etwas ältere Brugsch (Jg. 1878, habil. 1909) in den 1920er Jahren theoretische Fragestellungen der Konstitutionslehre und grundlegende Ausrichtungsfragen der Medizin zur wissenschaftlichen Profilierung. ${ }^{105}$ Als theoretischer Denker wurde Grote auch im Krisendiskurs rezipiert. ${ }^{106}$ Nicht nur bei den Konstitutionspathologen lässt sich beobachten, dass sie sich bereits vor dem Krisendiskurs zu grundlegenden Fragen oder kritisch zu Wesensfragen der Medizin geäußert hatten. Viele Partizipienten des Krisendiskurses, etwa Bier, Honigmann oder auch Aschner, nutzten die Krisendiskussion, um bereits früher vertretene Positionen breiter publik zu machen. ${ }^{107}$

\section{Fazit}

Sowohl Konstitutionslehre als auch das Stichwort «Krise der Medizin» boten gestaltungswilligen Medizinern ein Dach, um sich zu grundlegenden Ausrichtungsfragen der Medizin inklusive ihrer Forschungsepisteme zu äußern. Während dies innerhalb der Konstitutionslehre seit ihrer Begründung 1911 erfolgte und im Laufe der 1920er Jahre Impulse wie das Streben nach Ganzheitsbetrachtung und die Konzeption des Patienten als «psycho-physische Einheit» in die Konstitutionslehre einbrachte, bot der Krisendiskurs ab 1925

104 Krehl (Jg. 1861) emeritiert 1930; Kraus (Jg. 1858) em. 1927; His (Jg. 1863) em. 1932; Goldscheider (Jg. 1858) em. 1926, Klinikleitung bis 1933. Das gleiche gilt für August Bier (Jg. 1861) em. 1932.

105 Brugsch 1920; 1922; Brugsch/Lewy 1926. Brugsch 1928 gehört zum Krisendiskurs. Grote 1928 und 1930a gehören zum Krisendiskurs; außerdem zur Methodenkritik und Ausrichtung der Medizin Grote 1930b.

106 Honigmann 1928b, 178-179; auch schon Honigmann 1924, 18.

107 Für Bier siehe Lammel 1998, 193; für Honigmann siehe Knipper 2007, 370-73; für Aschner siehe oben. Muchs im Krisendiskurs stark rezipierte Bücher erschienen ebenfalls bereits vor dem Krisendiskurs, siehe oben. 
denselben Fragen unter seinem Teilbereich «wissenschaftliche Krise» Raum zur Diskussion. Konstitutionspathologen, die sich bereits vor 1925 berufen gefühlt hatten, aktiv zur Richtungsbestimmung «der Medizin» beizutragen, setzten dieses Engagement gerne auch im Rahmen des Krisendiskurses fort (etwa Krehl, Kraus, His, Goldscheider, Grote). In diesem unterschied sie nichts von anderen Diskursteilnehmern, die ebenfalls ab etwa 1925 ihre schon zuvor vertretenen Themen unter dem Krisenbegriff verhandelten (etwa Aschner, Bier, Honigmann).

Inhaltlich überschnitten sich die epistemologischen und medizinkritischen Forderungen der beiden Diskurse in vielen Punkten, insbesondere ihrer Kritik an Lokalismus, Mechanismus und Reduktionismus sowie die Forderung nach einer patientenzentrierten Perspektive. Allerdings bestanden die Antworten der Konstitutionslehre auf diese Kritikpunkte in der vom Krisendiskurs häufig gescholtenen Grundlagenforschung und insbesondere zur Anfangszeit naturwissenschaftlich ausgerichteten Methoden. Im Laufe der 1920er Jahre nahmen dann beide Diskurse gewisse übergreifende zeitgenössische Denkströmungen auf, was zu weiteren inhaltlichen Parallelen führte: Holismus, ${ }^{108}$ die Sehnsucht nach einer Synthese des medizinischen Denkens, ${ }^{109}$ sowie der Fokus auf das Individuum in seiner psycho-physischen Einheit. ${ }^{110}$ Diese Ideen schlugen sich in beiden Diskursen gleichermaBen nieder, ohne dem einen oder anderen exklusiv zuzugehören. ${ }^{111}$

Sieht man von Aschners Konstitutionstherapie ab, so wurde das Stichwort «Konstitution» im Krisendiskurs nur verhältnismäßig wenig erwähnt, obwohl das Thema von den jeweiligen Autoren meist als wichtige Fragestellung der zeitgenössischen Medizin gewürdigt wurde. Kurze, beiläufige Nennungen überwogen gegenüber ausführlichen Diskussionen. Dezidierte Positionierungen für oder gegen die Konstitutionslehre waren selten, häufiger wurde diese als eine grundlegende medizinische «Betrachtungsweise» (entsprechend eines Paradigmas) in eine historische Erzählung eingebettet, deren groben Züge alle Autoren teilten. Dabei wurde die Konstitutionslehre als gegenläufige Strömung zur streng naturwissenschaftlich ausgerichteten Medizin des späten 19. Jahrhunderts dargestellt, die sich vor dem Ersten Weltkrieg als Gegenentwurf zu Zellularpathologie, Lokalismus und Mecha-

108 Zum medizinischen Holismus der Weimarer Republik siehe Prüll 1998; Lawrence/Weisz 1998; Harrington 1999; Hau 2000.

109 Dazu etwa Zimmermann 1932; Brugsch 1933. Timmermann 1996, 56-58; Bothe 1991, 33.

110 Gadebusch Bondio/Michl 2012, 122-31; Gadebusch Bondio 2015; Metzger 2017, 285-291, 300.

111 Ausgeschlossen ist, dass die Konstitutionspathologen ihre holistische Rhetorik erst als Antwort auf die Krise wählten (vgl. Hofer 2003, 43), denn diese griffen den Holismus bereits weit vor 1925 auf, siehe etwa Brugsch 1920. 
nismus formiert und in einem grundlegenden Perspektivwechsel den einzelnen Patienten (wieder) in den Mittelpunkt der Medizin gerückt habe (insbes. Honigmann, Diepgen). ${ }^{112}$ Zwar griff diese Darstellung in weiten Teilen das Selbstkonzept der Konstitutionslehre als revolutionärer Gegenbewegung auf, das sich die junge Konstitutionslehre ab 1911 als Gründungsmythos gegeben hatte, ${ }^{113}$ doch betonte es einseitig deren holistisch orientierte Fortentwicklungen der 1920er Jahre, während zentrale konstitutionspathologische Arbeitsgebiete wie etwa die keineswegs patientennahe Grundlagenforschung, mit selbstverständlichem Gebrauch naturwissenschaftlicher Methoden und Laborforschung, ausgeblendet wurden.

Zeichneten die Beiträger zum Krisendiskurs solchermaßen alle ein ähnliches Bild der historischen Entwicklungen, rangierten ihre darin enthaltenen Verknüpfungen von Konstitutionslehre und wissenschaftlicher Krise von symptomatisch (Sauerbruch, Siegerist) bis kausal (Krehl). Durch die historisierende Darstellung erschien die Moderne Konstitutionslehre bei diesen Autoren mehr als ein Phänomen der jüngeren Vergangenheit als der Zukunft; Potenzial zu Veränderung wurde ihr dadurch kaum zugesprochen.

Nur wenige Autoren diskutierten gegenwartsbezogen, was eine Beschäftigung mit Konstitution in der wissenschaftlichen Krise anzubieten habe. Ihr Urteil fiel gemischt aus: Ablehnung (Bier), Skepsis gepaart mit der Forderung nach mehr therapeutischer Forschung (Honigmann) und enthusiastische Bewerbung (Much). Auffällig ist, dass das Thema «Konstitution» ausschließlich von den Autoren als Chance in der Krise dargestellt wurde, die ihre eigene konstitutionsbezogene Lehre propagierten: Much seine spezielle Spielart der Konstitutionslehre, Brugsch seine Weiterentwicklung zum «Personalismus» und Aschner seine humoralpathologische «Konstitutionstherapie».114 Als einziger dieser drei verortete sich Much selbst innerhalb der Modernen Konstitutionslehre, allerdings ohne seine Veröffentlichungen unter das Schlagwort der «Krise» zu stellen. Anders als Brugsch setzte sich Aschner zudem auch methodisch stark von der Modernen Konstitutionslehre ab.

Dieser Befund zeigt, dass die Konstitutionslehre im Sinne der Modernen Konstitutionslehre nur selten als Antwort auf die Krise der Medizin gehan-

112 Genau dieses Sinnbild eines Pendels, das von naturwissenschaftlicher Reduktion im späten 19. Jahrhundert zum patientenzentrierten Blick der Konstitutionslehre zurückschlug, sollte in den nächsten sechzig Jahren nachhaltig medizinhistorische Darstellungen der Konstitutionslehre prägen - nach 1945 insbesondere vermittelt durch Diepgens «Geschichte der Medizin» (1949-1955), siehe auch Probst 1982. Ebenfalls kritisch zu dieser Sichtweise: Timmermann 2001, 727-28.

113 Metzger 2016, insbes. 209-10, 213-14, 218-19, 237-38.

114 Jaensch mit seiner speziellen konstitutionspathologischen Richtung zähle ich hier nicht auf, da er in seiner Veröffentlichung (Jaensch 1933) die Krise als überwunden darstellt. 
delt wurde. ${ }^{115}$ Konstitutionsbezogene Ansätze wie Personalismus und Konstitutionstherapie - so unterschiedlich sie sind - hingegen sehr viel häufiger. Wirklichen Nachhall im Krisendiskurs fand von diesen Ansätzen aber nur Aschner, dessen Konstitutionstherapie intensiv und positiv rezipiert wurde.

Gerade die große Anzahl historisierender Darstellungen der Konstitutionslehre im Krisendiskurs unterstreicht den Eindruck, dass die Konstitutionslehre in den Jahren 1925-1933 ihren Nimbus als Hoffnungsträgerin einer Medizin der Zukunft weitgehend verloren hatte. Kaum ein Autor sprach ihr noch Erneuerungspotenzial für Forschungsepisteme und praktische Ausübung der zeitgenössischen Medizin zu; auffälligerweise gilt dies gerade auch für die Autoren, die in der Frühzeit der Konstitutionslehre maßgeblich an deren Konzeption beteiligt gewesen waren. Fünfzehn bis zwanzig Jahre konstitutionspathologischer Grundlagenforschung scheint bei diesen Autoren zu einiger Desillusionierung bezüglich deren Möglichkeiten geführt zu haben, zumal die Konstitutionslehre auf den für die Krisenautoren so wichtigen Gebieten der medizinischen Praxis und Therapie kaum Verbesserungen erwirkt hatte. Aus dem vormals ambitionierten Forschungsprogramm zur Revolutionierung der Medizin war im Krisendiskurs somit ein Phänomen der (jüngeren) Vergangenheit geworden, besprochen in historischen Abrissen oder überholt durch eigene Lehrgebäude der Krisenautoren.

\section{Bibliographie}

Aschner, Bernhard, Die Blutdrüsenerkrankungen des Weibes und ihre Beziehungen zur Gynäkologie und Geburtshilfe (Wiesbaden 1918).

Aschner, Bernhard, «Die praktische Bedeutung der Lehre vom Habitus und die Renaissance der Humoralpathologie als therapeutische Konsequenz der Konstitutionslehre», in: Wiener Klinische Wochenschrift 35 (1922) 73-77, 107-09.

Aschner, Bernhard, «Konstitutionslehre und Humoralpathologie, mit besonderer Berücksichtigung des weiblichen Organismus», in: Deutsche Medizinische Wochenschrift 50 (1924) 1277-80.

Aschner, Bernhard, Die Krise der Medizin. Konstitutionstherapie als Ausweg (Stuttgart, Leipzig, Zürich 1928).

Aschner, Bernhard, Heilerfolge der Konstitutionstherapie bei weiblichen Geisteskranken insbesondere bei Schizophrenie (Stuttgart, Leipzig 1933).

115 Dazu siehe oben. Klasen 1984; Timmermann 1996; 1999; 2001; Hofer 2007. 
Baader, Gerhard, «Eugenische Programme in der sozialistischen Parteienlandschaft in Deutschland und Österreich im Vergleich», in: Derselbe et al. (Hrsg.), Eugenik in Österreich. Biopolitische Strukturen von 1900-1945 (Wien 2007) 66-139.

Bauer, Julius, «Rez. 'Die Konstitution der Frau und ihre Beziehungen zur Geburtshilfe und Gynäkologie. Von Bernhard Aschner'», in: Klinische Wochenschrift 3 (1924) 1690.

Bier, August, «Wesen und Grundlagen der Heilkunde. 2. Teil: Hippokratismus (Schluß). Dritter Abschnitt: Die Physis», in: Münchener Medizinische Wochenschrift 78 (1931) 408-11.

Borak, J., «Zur konservativen Therapie der Uterusblutungen. Bemerkungen zu Aschners Aufsatz: Konservative und operative Therapie der Gebärmutterblutungen», in: Wiener Medizinische Wochenschrift 76 (1926) 404-06 (mit Erwiderung Aschners ebd. 406-10).

Bothe, Detlef (1991): Neue Deutsche Heilkunde 1933-1945. Dargestellt anhand der Zeitschrift «Hippokrates» und der Entwicklung der volksheilkundlichen Laienbewegung, Abhandlungen zur Geschichte der Medizin und der Naturwissenschaften 62 (Husum 1991).

Brugsch, Theodor, «Personalismus», in: Berliner klinische Wochenschrift 57 (1920) 317-18.

Brugsch, Theodor, «Ziel und Wege der Konstitutionsforschung oder die Personallehre», in: Medizinische Klinik 18 (1922) 1082-83.

Brugsch, Theodor, «Die Klinik», in: Grundlagen und Ziele der Medizin der Gegenwart. Fünf Vorträge, gehalten im Winter 1927/28 von Theodor Brugsch, Curt Elze, Luois R. Grote, E. Liek, W. Mayer-Gross (Leipzig 1928) 51-71.

Brugsch, Theodor (Hrsg.), Einheitsbestrebungen in der Medizin. Kongreß zur Förderung medizinischer Synthese und ärztlicher Weltanschauung. Verhandlungsbericht der zweiten zwischenstaatlichen Tagung in Marienbad 14.-17. September 1932 (Dresden, Leipzig 1933).

Brugsch, Theodor/Lewy, Friedrich H. (Hrsg.), Die Biologie der Person. Ein Handbuch der allgemeinen und speziellen Konstitutionslehre. Bd. I: Allgemeiner Teil der Personallehre. Insg. 4 Bde. 1926-1931 (Berlin 1926).

Brunk-Loch, Sibylle, Bernhard Aschner (1883-1960). Sein Weg von der Endokrinologie zur Konstitutionstherapie (Diss. med. Univ. Mainz 1995).

Bumke, Oswald, Eine Krisis der Medizin. Rede gehalten bei der Übernahme des Rektorats am 24. November 1928 (München 1929).

Diepgen, Paul, «Krankheitswesen und Krankheitsursache in der spekulativen Pathologie des 19. Jahrhunderts», in: Archiv für Geschichte der Medizin 18 (1926) 302-27.

Diepgen, Paul, «Die Grundlagen der Medizin im 19. Jahrhundert und ihre gegenwärtige Krise», in: Deutsche Medizinische Wochenschrift 54 (1928) 2171-75.

Diepgen, Paul, «Die Lehre von der Konstitution in der vitalistischen Medizin», in: Klinische Wochenschrift 12 (1933) 30-32.

Diepgen, Paul, «Zur Geschichte der Lehre von der Konstitution», in: Walther Jaensch (Hrsg.), Konstitutions- und Erbbiologie in der Praxis der Medizin. Vorträge eines Internationalen Fortbildungskurses in der Berliner Akademie für ärztliche Fortbildung im Frühjahr 1934 (Leipzig 1934) 1-15.

Diepgen, Paul, Geschichte der Medizin. Die historische Entwicklung der Heilkunde und des ärztlichen Lebens. II. Band, 2. Hälfte: Die Medizin vom Beginn der Zel- 
lularpathologie bis zu den Anfängen der modernen Konstitutionslehre (etwa 1858-1900). Mit einem Ausblick auf die Entwicklung der Heilkunde in den letzten 50 Jahren (Berlin 1955).

Doms, Misia Sophie, «August Biers Aufsatz 'Wie sollen wir uns zu der Homöopathie stellen?' (1925) und die nachfolgende Diskussion um die Homöopathie in der deutschen Ärzteschaft», in: Medizin Gesellschaft und Geschichte 23 (2005) 24382 .

Felsch, Philipp, «Ganzheit und ihre Apparaturen. Konstitutionelle Medizin um 1900», in: Cornelius Borck et al. (Hrsg.), Maß und Eigensinn. Studien im Anschlu $\beta$ an Georges Canguilhem (München 2005) 135-56.

Fischer, Isidor/Voswinckel, Peter, s.v. «Aschner, Bernhard», in: Biographisches Lexikon der hervorragenden Ärzte der letzten fünfzig Jahre. Nachträge und Ergänzungen, Bd. III (Hildesheim 2002a) 49.

Fischer, Isidor/Voswinckel, Peter: s.v. «Bier, August», in: Biographisches Lexikon der hervorragenden Ärzte der letzten fünfzig Jahre. Nachträge und Ergänzungen, Bd. III (Hildesheim 2002b) 123-24.

Gadebusch Bondio, Mariacarla, «'Das Individuum - eine Abweichung' ... und das Unbehagen der Wissenschaft», in: Eva Brinkschulte/Mariacarla Gadebusch Bondio (Hrsg.), Norm als Zwang, Pflicht und Traum. Normierende versus individualisierende Bestrebungen in der Medizin, Medizingeschichte im Kontext 19 (Frankfurt/Main 2015) 19-50.

Gadebusch Bondio, Mariacarla/Michl, Susanne, «Von der Medikalisierung des Humanen. Das Individuelle als Herausforderung», in: Mariacarla Gadebusch Bondio et al. (Hrsg.), Konzepte des Humanen. Ethische und kulturelle Herausforderungen (Freiburg 2012) 117-38.

Geiger, Karin, «'Krise' - zwischen Schlüsselbegriff und Schlagwort. Zum Diskurs über eine 'Krise der Medizin' in der Weimarer Republik», in: Medizinhistorisches Journal 45 (2010) 368-410.

Gesellschaft der Ärzte in Wien, «Bericht der Sitzung vom 22. Mai 1931», in: Wiener Medizinische Wochenschrift 81 (1931a) 826-29.

Gesellschaft der Ärzte in Wien, «Bericht der Sitzung vom 12. Juni 1931», in: Wiener Medizinische Wochenschrift 81 (1931b) 921-25.

Goldscheider, Alfred, «Zeit- und Streitfragen der Heilkunst: I. Sauerbruchs Intuition; II. Liek: Der Arzt und seine Sendung; III. Das Wesen des Kurpfuschertums und der ärztlichen Wissenschaft», in: Deutsche Medizinische Wochenschrift 53 (1927) 203-05; 244-47; 289-91; 331-33; 376-77.

Grote, Louis R., «Der funktionelle Gedanke», in: Grundlagen und Ziele der Medizin der Gegenwart. Fünf Vorträge, gehalten im Winter 1927/28 von Theodor Brugsch, Curt Elze, Luois R. Grote, E. Liek, W. Mayer-Gross (Leipzig 1928) 2350 .

Grote, Louis R., Grundlagen ärztlicher Betrachtung. Einführung in begriffliche und konstitutionspathologische Fragen der Klinik für Studierende und Ärzte (Berlin 1921).

Grote, Louis R., «Die innere Entwicklung der modernen Medizin und ihre praktische Auswirkung», in: Blätter des deutschen Roten Kreuzes 9 (1930a) 644-57.

Grote, Louis R., «Krankheitslehre und Persönlichkeit», in: Natur und Museum 60 (1930b) 49-64. 
Gruber, Georg Benno: «Zur angeblichen Krisis der Medizin», in: Wiener klinische Wochenschrift 46 (1933), 801-07.

Hahn, Marcus, Gottfried Benn und das Wissen der Moderne. 2 Bde. (Göttingen 2011).

Harrington, Anne, Reenchanted Science. Holism in German culture from Wilhelm II to Hitler (Princeton/NJ 1999).

Hau, Michael, «The Holistic Gaze in German Medicine, 1890-1930», in: Bulletin of the History of Medicine 74 (2000) 495-524.

Hau, Michael, The Cult of Health and Beauty in Germany. A social history 18901930 (Chicago 2003).

Hau, Michael, «The Humane Expert. The crisis of modern medicine during the Weimar Republic», in: Elke Kurz-Milcke/Gerd Gigerenzer (Hrsg.), Experts in Science and Society (New York 2004) 105-22.

Hau, Michael, «Constitutional Therapy and Clinical Racial Hygiene in Weimar and Nazi Germany», in: Journal of the History of Medicine and Allied Sciences 71 (2015) 115-43.

His, Wilhelm, «Geschichtliches und Diathesen in der Inneren Medizin», in: Verhandlungen des Deutschen Kongresses für Innere Medizin 28 (1911) 15-35.

His, Wilhelm, «Die Krise in der Medizin», in: Die Woche (1930) 789-90.

His, Wilhelm, «Die Krise in der Medizin», in: Die Ernte. Halbmonatsschrift für Politik und Allgemeines 13 (1932) 25-28.

Hofer, Veronika, «Positionen und Posen eines Experten. Der Konstitutionsforscher Julius Bauer (1887-1973) und die Eugenik in der Wiener Zwischenkriegszeit», in: Gerhard Baader et al. (Hrsg.), Eugenik in Österreich. Biopolitische Strukturen von 1900-1945 (Wien 2007) 31-65.

Honigmann, Georg, Über medizinische Systematik (Leipzig 1924).

Honigmann, Georg, «Die Problematik der heutigen Medizin», in: Die medizinische Welt 1 (1927) 1216-18, 1261-63, 1375-77.

Honigmann, Georg, «Einheitsbestrebungen der Gegenwartsmedizin». in: Hippokrates 1 (1928a) 3-15.

Honigmann, Georg, «Die Krise der Medizin in der literarischen Beleuchtung der letzten Jahre». in: Hippokrates 1 (1928b) 170-180.

Huismans, Laurenz, «Reaktion und Reform in der Medizin», in: Hochland 26 (1929) 464-75.

Jaehn, Thomas, Der Medizinhistoriker Paul Diepgen (1878-1966). Eine Untersuchung zu methodologischen und zeitgeschichtlichen Problemen und Einflüssen im Werk Paul Diepgens unter besonderer Berücksichtigung seiner persönlichen Rolle in Lehre, Wissenschaftspolitik und Wissenschaftsorganisation während des Dritten Reiches (Diss. med. HU Berlin 1991).

Jaensch, Walther, «Konstitutionsmedizin und Kulturprobleme in der Krise der Gegenwart», in: Deutsche Medizinische Wochenschrift 59 (1933) 1088-90.

Jehs, Michael, Erwin Liek. Weltanschauung und standespolitische Einstellung im Spiegel seiner Schriften (Frankfurt am Main 1994; zugl. Diss. med. Univ. Kiel 1992).

Klasen, Eva-Maria, Die Diskussion über eine «Krise» der Medizin in Deutschland zwischen 1925 und 1935 (Diss. med. Univ. Mainz 1984).

Knipper, Michael, «Medizin zwischen Wissenschaft und Heilkunst? Der Gießener Internist und Medizinhistoriker Georg G. Honigmann (1863-1930) und die 'Krise 
der Medizin' zur Zeit der Weimarer Republik», in: Ulrike Enke (Hrsg.), Die Medizinische Fakultät der Universität Gießen. Institutionen, Akteure und Ereignisse von der Gründung 1607 bis ins 20. Jahrhundert (Stuttgart 2007) 369-94.

Kölch, Michael Gregor, Theorie und Praxis der Kinder- und Jugendpsychiatrie in Berlin 1920-1935. Die Diagnose «Psychopathie» im Spannungsfeld von Psychiatrie, Individualpsychologie und Politik (Diss. med. FU Berlin 2006).

Kraus, Friedrich, «Über Grundlagenkrise in der medizinischen Wissenschaft», in: Archiv für klinische Chirurgie 164 (1931) 1-4.

Kraus, Friedrich, «Grundlagenkrise in der medizinischen Theoriebildung?», in: Zeitschrift für ärztliche Fortbildung 29 (1932) 90-92.

Krehl, Ludolf, «Eröffnungsrede», in: Verhandlungen des Deutschen Kongresses für Innere Medizin 28 (1911) 3-11.

Krehl, Ludolf, Pathologische Physiologie. Zwölfte Auflage (Leipzig 1923).

Krehl, Ludolf, «Ueber Standpunkte in der inneren Medizin», in: Münchener Medizinische Wochenschrift 73 (1926) 1547-52.

Krügel, Rainer, Friedrich Martius und der konstitutionelle Gedanke (Frankfurt/ Main 1984).

Lammel, Hans-Uwe, «Chirurgie und Nationalsozialismus am Beispiel der Berliner Chirurgischen Universitätsklinik in der Ziegelstraße», in: Wolfram Fischer et al. (Hrsg.), Exodus von Wissenschaften aus Berlin. Fragestellungen - Ergebnisse Desiderate. Entwicklungen vor und nach 1933, Akademie der Wissenschaften zu Berlin. Forschungsbericht 7 (Berlin, New York 1994) 568-91.

Lammel, Hans-Uwe, «August Bier und der dritte Humanismus». in: Klaus-Dietrich Fischer et al. (Hrsg.), Text and Tradition. Studies in ancient medicine and ist transmission. Presented to Jutta Kollesch (Leiden 1998), 175-202.

Lawrence, Christopher/Weisz, George (Hrsg.), Greater Than the Parts. Holism in biomedicine, 1920-1950 (New York 1998).

Lehfeldt, Hans, «Renaissance-Bewegung in der Medizin», in: Das Tagebuch 8 (1927) 660-64.

Liek, Erwin, Der Arzt und seine Sendung. Gedanken eines Ketzers (München 1926).

Martius, Friedrich, Konstitution und Vererbung in ihren Beziehungen zur Pathologie (Berlin 1914).

Martius, Friedrich, «Friedrich Martius», in: Louis R. Grote (Hrsg.), Die Medizin der Gegenwart in Selbstdarstellungen. Bd. 1 (Leipzig 1923) 105-40.

Mayer, Ernst, Die Krisis des deutschen Ärztestandes. Eine soziologische Untersuchung (Berlin 1924).

Metzger, Nadine, «'Auf strengster wissenschaftlicher Grundlage'. Die Etablierungsphase der modernen Konstitutionslehre 1911 bis 1921», in: Medizinhistorisches Journal 51 (2016) 209-45.

Metzger, Nadine, «'Es sind noch große Forschungserträge zu erhoffen.' Entwicklungen der Konstitutionslehre in den 1920er Jahren», in: Medizinhistorisches Journal 52 (2017) 270-307.

Moses, Julius, «Die Krise der Medizin», in: Biologische Heilkunst 10 (1929) 804-05, 832-33.

Much, Hans, Hippokrates der Grosse (Stuttgart 1926).

Much, Hans, Das Wesen der Heilkunst. Grundlagen einer Philosophie der Medizin (Darmstadt 1928). 
Much, Hans, Steht die scholastische Medizin vor einem unvermeidlichen Bankerott? (Leipzig 1931).

Pfaundler, Meinhard, «Diathesen in der Kinderheilkunde», in: Verhandlungen des Deutschen Kongresses für Innere Medizin 28 (1911) 36-85.

Probst, Johannes, Zur Entwicklung der Konstitutionslehre zwischen 1911 und 1980 (Diss. med. Freiburg 1982).

Prüll, Cay-Rüdiger, «Holism and German Pathology (1914-1933)», in: Christopher Lawrence/George Weisz (Hrsg.), Greater Than the Parts. Holism in biomedicine, 1920-1950 (New York 1998) 46-67.

Prüll, Cay-Rüdiger, Medizin am Toten oder am Lebenden? Pathologie in Berlin und in London, 1900-1945 (Basel 2003).

Roelcke, Volker, «'Krise der Medizin' - Modelle der Reform. Zur Frühgeschichte von Psychotherapie und Sozialwissenschaften in der Medizin», in: Psychotherapeut 61 (2016) 237-242.

Sauerbruch, Ferdinand «Heilkunst und Naturwissenschaft», in: Die Naturwissenschaften 14 (1926) 1081-90.

Schmidt, P[eter], «Krise der Medizin?» in: Querschnitt 9 (1929) 318-20.

Schmiedebach, Hans-Peter, «Der wahre Arzt und das Wunder der Heilkunde. Erwin Lieks ärztlich-heilkundliche Ganzheitsideen», in: Heinz-Harald Abholz (Hrsg.), Der ganze Mensch und die Medizin (Hamburg 1989) 33-53.

Schmiedebach, Hans-Peter, «Medizin und Ärzte in der Krise. Ein Blick in die 1920er Jahre», in: Oliver Erens (Hrsg.), Geschichte(n) der Medizin. Resümee wichtiger medizinhistorischer Beiträge aus 15 Jahren Ärzteblatt Baden-Württemberg (Stuttgart 2014) 119-27.

Schulze-Rath, Renate, Hans Much (1880-1932). Bakteriologe und Schriftsteller (Diss. med. Univ. Mainz 1993).

Sigerist, Henry E., «Das Bild des Menschen in der modernen Medizin», in: Neue Blätter für den Sozialismus 1 (1930) 97-106.

Timmermann, Carsten, Concepts of the Human Constitution in Weimar Medicine, 1918-1933 (MA thesis Univ. Manchester 1996).

Timmermann, Carsten, Weimar Medical Culture. Doctors, healers, and the Crisis of Medicine in interwar Germany, 1918-1933 (PhD thesis Univ. Manchester 1999).

Timmermann, Carsten, «Constitutional Medicine, Neoromanticism, and the Politics of Antimechanism in Interwar Germany», in: Bulletin of the History of Medicine 75 (2001) 717-39.

U.T., «Krisis in der modernen Medizin» \& B. Aschner, «Erwiderung», in: Biologische Heilkunst 9 (1928) 565-66 und 654.

Weindling, Paul, Health, Race and German Politics Between National Unification and Nazism 1870-1945 (Cambridge 1989).

Wirtz, Rainer, Leben und Werk des Hamburger Arztes, Forschers und Schriftstellers Hans Much (1880-1932) unter besonderer Berücksichtigung seiner medizintheoretischen Schriften (Herzogenrath 1991, zugl. Diss. med. Aachen 1991).

Zimmermann, Heinz, «Theoretische Biologie und Medizin der Gegenwart», in: Klinische Wochenschrift 11 (1932) 819-24. 